\title{
Keys to the flesh flies of Thailand, with description of a new species of Robineauella Enderlein (Diptera: Sarcophagidae)
}

\author{
Hiromu Kurahashi ${ }^{1)}$ and Tarinee Chaiwong $^{2)}$ \\ 1) Department of Medical Entomology, National Institute of Infectious Diseases, \\ Toyama 1-23-1, Shinjuku-ku, Tokyo 162-8640, Japan \\ 2) College of Medicine and Public Health, Ubon Ratchathani University, \\ Ubon Ratchathani 34190, Thailand
}

(Received: 9 November 2012; Accepted: 8 January 2013)

\begin{abstract}
The collection of Sarcophagidae made by the first author during the surveys on flies of medical and forensic importance in Thailand from 1975 to 2011 is dealt with. A total of 29 genera and 83 species of the flesh flies are listed. Twelve species including Miltogramma iberica Villeneuve, 1912 are newly recorded from Thailand. One new species of the genus Robineauella Enderlein is described and illustrated, which is similar to Robineauella coei Rohdendorf, 1966 from Nepal and the widespread species R. pseudoscoparia (Kramer, 1911) in general appearance. Revised identification keys to the Thai species are provided including a new and newly recorded ones.
\end{abstract}

Key words: flesh flies, keys, new species, Robineauella, Sarcophagidae, Thailand

\section{INTRODUCTION}

During the field survey of the "Zoogeographical Studies on the Medically Important Diptera in Southeast Asia", the first author and the members of the field survey made an excellent collection of the flesh flies in 1975. After examination of these collections, Tumrasvin and Kano (1979) reported 19 genera and 48 species of the family Sarcophagidae from Thailand although this would be covered only a part of whole fauna, indeed. Recently the present authors tried to collect flies in different localities and various habitats in order to make a good series of reference collections for understanding the entire fly fauna of Thailand. The collected samples were mounted with insect pins in good order. All specimens were sorted into subfamilies. In the course of our taxonomical studies, we also had opportunities to examine the preserved materials in the museums whose names are indicated in ( ) in the checklist of species below. We have examined a total of 29 genera and 83 species of Thai flesh flies or Sarcophagidae. Of 83 species, we found one species new to science, which belongs to the genus Robineauella Enderlein. The new species is similar to Robineauella coei Rohdendorf, 1966 from Nepal and the widespread species R. pseudoscoparia (Kramer, 1911) in general appearance, but easily can be distinguished from these two previously known species by the characteristic shape of male genitalia having the truncate apex of juxta in lateral view and cercus without spinous setulae along anteior margin. We found 13 species never been recorded from Thailand before. New country records are marked with an asterisk $\left({ }^{*}\right)$. The previous keys (Tumrasvin and Kano, 1979) to the Thai species are re- vised to include the newly recorded ones and rearranged by the modern systematics.

Identified specimens are mostly preserved in the Mueum of Nature and Science, Tsukuba (NSMT). Some were shared and transferred into the collection of several museums such as the Bishoip Museum, Honolulu (BPBM); Carnegie Museum of Natural History, Pittsburgh (CMNH); Natural History Museum, London $(\mathrm{BMNH})$. Names of museum are indicated in [ $>$ ] in the list of species below.

We tried to analyze the flesh fly fauna of Thailand. Most fly species seem to be so-called Oriental elements (53\%) and endemic or only known in Thailand (23\%), and then Palaearctic (20\%), Pantropical or cosmopoli$\tan (3 \%)$ and Afrtotropical ones (1\%). Rather high endemicity (19 species, $23 \%$ ) seems to be cahracteristics of sarcophagid fauna in Thailand.

Abbreviations for institutions housing specimens are as follows: BPBM, Bishop Museum, Honolulu; CAS, California Academy of Sciences, San Francisco; DPCM, Department of Parasitology, Chiang Mai University, Chiang Mai; CMPH, College of Medicine and Public Health, Ubon Ratchathani University, Ubon Ratchathani; IDD, International Department of Dipterology, Tokyo; NIID, Reference Museum, Department of Medical Entomology, National Institute of Infectious Diseases, Tokyo; IRScNB, Zoological Institute, Louvain; MUTM, Faculty of Tropical Medicine, Mahidol University, Bangkok; NSMT, National Museum of Nature and Science, Tsukuba; PCHB, Personal collection of Hans Bänziger, Chiang Mai University; SMNH, Swedish Museum of Natural History, Stockholm; UM, University of Malaya, Kuala Lumpur; ZMUC, Zoological Museum, University of Copenhagen; TMDU, Tokyo 
Medical and Dental University. All fly specimens of TMDU were already transferred to NSMT for a permanent preservation. Locality names of specimens examined are cited as shown on the label of specimen. Terminology mainly follows Senior-White et al (1940) and measurement of frons was made in a similar manner to Fan (1965). Frons index is the same as Fan's index of frons.

\section{Practical Keys}

\section{Key to the subfamilies of Thai Sarcophagidae}

1. Hind coxa hairy on posterior surface; $n$, two strong primary bristles, two smaller subprimary bristles; sternites 3 to 4 fully exposed and overlapping ventral margins of corresponding tergite ........ .. Subfamily SARCOPHAGINAE

- Hind coxa bare on posterior surface; $n 2$; sternites 3 to 4 , more or less concealed by ventral margin of corresponding tergite

2. Arista plumose or pubescent; hypopygium large; $\mathrm{GS}_{1}$ amalgamated with tergite 6 with row of erect marginal bristles [No record from Thailand] ……... …………..... Subfamily PARAMACRONYCHIINE

- Arista bare or pubescent; hypopygium small; tergite 6 free, not amalgamated with $\mathrm{GS}_{1}$ Subfamily MILTGOGRAMMATINAE

Subfamily M I L T O G R A M M A T I N A E

\section{Key to the tribes and genera}

1. Mesothoracic spiracle open, without operculum; legs long, bristly, with elongated claws in $0^{7}$; parafacial and gena broad, hairy; oral margin in profil not projecting forward; body covered with pale dusting, sometimes having long ovipositor exsert- ed; length 5-10 mm [No record from Thailand] ..... ......... Tribe Macronychiini, Macronychia Rondani

- Mesothoracic spiracle covered with operculum; legs short, usually with short claws, sometimes with elongated ones in $0^{\top}$ of Senotainia; but in this case epistomal margin in profil angulated, and body length not more than $6 \mathrm{~mm}$; colouration variable, often with pale spots in contrast with black bands and spots . .2

2. Head hemi-spherical; eyes very large; numerous fine proclinate and 1-2 strong reclinate fronto-orbital bristles (ors) present (Kurahashi, 1970: 95, Fig. 1C)

....... Tribe Amobiini, Amobia Robineau-Desvoidy

- Head subquadrate; eyes usually normal in size; 0-4 (proclinate) $+1-2$ (reclinate) ors (Kurahashi, 1970: 95, Fig. 1A \& B) …. Tribe Miltgrammatini 3

-- Head conical: eyes very large; ors $2+3$ (Kurahashi, 1970: 95, Fig. 1D)

. Tribe Metopiini, Metopia Meigen

3. Claws and pulvilli long in both sexes (Kurahashi, 1970: 97, Fig. 2A), sometimes normal in 우 abdominal tergites with three spots on dorsum ........... .......... Senotainia Macquart, S. navigatrix (Meijere)

- Claws and pulvilli normal in both sexes (Kurahashi, 1970: 97, Fig 2B); abdominal tergites with dark marginal band, or tessellate ………………...... 4

-- Claws and pulvilli normal in both sexes (Kurahashi, 1970: 97, Fig. 2B); abdominal tergites with three dark spots ...

.... Hoplacephala Macquart, ${ }^{*} H$. linearis Villeneuve

4. Vibrissa present on vibrissal angle high above oral margin .................... Protomiltogramma Townsend - Vibrissa absent ........................ Miltogramma Meigen

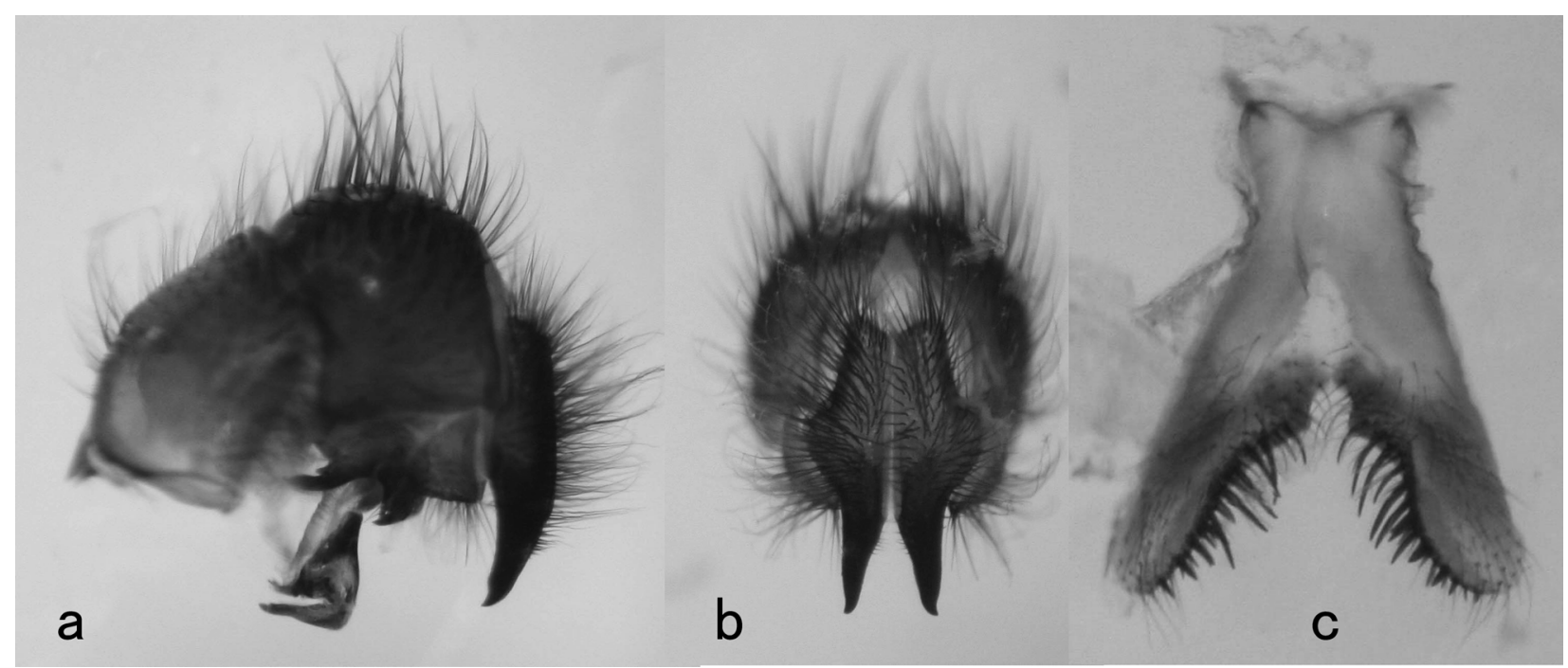

Fig. 1. Robineauella thailandica sp. nov., male copulatory aparatus. - a, male terminalia, lateral view; b, epandirum, cercus and surstylus, caudal view; c, fifth sternite, ventral view. 


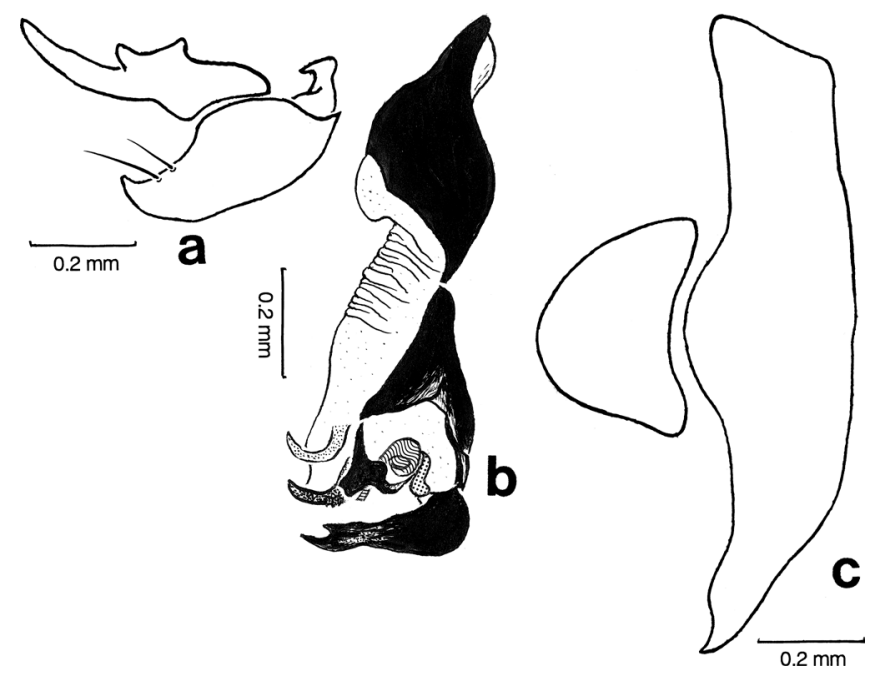

Fig. 2. Robineauella thailandica sp. nov., male genitalia. - a, pregonite and postgonite, lateral view; b, aedeagus, lateral view; c, surstylus and cercus, lateral view.

\section{Key to the species of Amobia}

1. Basicosta yellow; thoracic dorsum with distinct three longitudinal stripes ... * A. auriceps (Baranov)

- Basicosta blackish; three longitudinal stripes indistinct *A. quatei Kurahashi

\section{Key to the species of Protomiltogramma}

1. Tergites 3-4 each with three dark spots postriorly; notopleuron bare ……... P. macularis (Wiedemann)

- Tergites 3-4 distinctly marginal banded; notopleuron hairy ... 2

2. Third antennal segment $\left(\mathrm{AS}_{3}\right)$ elongate, slightly more than $5 \mathrm{X}$ as long as second antennal segment $\left(\mathrm{AS}_{2}\right)$; postgena clothed with black hairs; tergite 5 with well developed tuft of bristles on posteroventral margin in $0^{\top}$...P. komi Kurahashi \& Chaiwong

- $\mathrm{AS}_{3}$ moderate in length, slightly more than $2.5 \mathrm{X}$ as long as $\mathrm{AS}_{2}$; postgena clothed with white hairs; tergite 5 without distinct tuft of bristles, but several bristles present along posteroventral margin in $\sigma^{7}$ P. kabkaewae Kurahashi \& Chaiwong

\section{Key to the species of Miltogramma}

1. $\mathrm{AS}_{3}$ largely orange yellow, especialy on inner surface ………………… M. angustifrons (Townsend)

- $\mathrm{AS}_{3}$ entirely fuscous brown to black * M. iberica Villeneuve

\section{Key to the species of Metopia}

1. Basicosta black 2

- Basicosta yellow 3

2. Vein $\mathrm{R}_{1}$ bare *M. sauteri (Townsend)

- Vein $\mathrm{R}_{1}$ setulose ${ }^{*}$ M. nudibasis (Malloch)
3. Tergite $1+2$ with erect, sometimes decumbent median $m b$; silvery pollinosed part of parafrontal abruptly demarcated from posterior dark portion

- Tergite $1+2$ without median $m b$; silvery pollinosed part of parafrontal with gradual transition to posterior dark portion 5

4. Anterior part of frontal stripe distinct to lunule; row of frontal bristles continuous and not interrupted in $\sigma^{\top}$ (Povolný \& Verves, 1997: Fig. 86) ........ ... M. tschernovae Rohdendorf

- Anterior part of frontal stripe linear; row of frontal bristles less distinct, completely interupted in $0^{7}$ (Povolný \& Verves, 1997: Fig. 88) *M. argyrocephala (Meigen)

5. Alar and thoracic squamae whitish; parafrontal and parafacial silver pollinose in 우; body silvergrey pollinose (Povolný \& Verves, 1997, Fig. 87) .... *M. argentata Macquart

- Alar and thoracic squamae yellowish; parafrontal and parafacial yellowish-silver to golden pollinose in ㅇ; body yellowish to golden pollinose ……….... 6

6. Frontal stripe moderately broad, parallel-sided even on anterior part of frons in $\sigma^{7}$; cross vein $\mathrm{m}-\mathrm{m}$ curved, sigmoid; vein $\mathrm{R}_{1}$ bare in + ; apical section of vein $\mathrm{M}_{1+2}$ from $\mathrm{m}-\mathrm{m}$ to bend shorter than basal section in 우

M. yunnanica Chao \& Zhang

- Frontal section very narrow, nearly linear in $\sigma^{\top}$; cross vein $\mathrm{m}$-m straight in 우; vein $\mathrm{R}_{1}$ usually with 1-2 black setulae in 우 apical section of vein $\mathrm{M}_{1+2}$ from $\mathrm{m}-\mathrm{m}$ to bend subequal to basal section in + + M. suifenhoensis Fan 


\section{Subfamily S A R C O P H A G I N A E}

\section{Key to tribes and genera}

1. Arista pubescent, hairs not exceed width of arista; st $1+1$; body densely silver-grey dusted; abdomen weakly tessellated (Tumrasvin and Kano, 1979: Fig. 48) …….... Leucomyia Brauer \& Bergenstamm, L. alba (Schiner)

- Arista long plumose, plumose hairs longer than width of arista; st $1+1+1$, rarely median one weakly developed or absent in Helicophagella melanura; body more or less darkened, grey pollinose, abdominal pollinosity produce irregular checkered patterns or tessallation, but sometimes dark spots and median stripe in of of Blaesoxipha spp. ............ 2

2. Row of ori nearly straight in dorsal view [ No record from Thailand] ....

......... Tribe Raviniini, Ravinia Robineau-Desvoidy

- Row of ori distinctly diverging at lunule ................. 3

3. Presutural $a c$ strong, usually arranged in distinct row, from anterior to posterior part of presutural area: $\sigma^{7}$ cercus distinctly bent back; 우 sternites $7-8$ fussed and often form sclerotized ovipositor shovel-shaped, blade-like, or recurving beneath abdomen; SPAP vestigial, but with pair or pairs of setulae; male sternite 5 without spine-like bristles along inner margins of lateral lobes...

- Tribe Protodexiini, Blaesoxipha Loew

- Presutural ac absent, at most relatively weakly developed ac present on anterior and/or posterior prescutelar areas; $\sigma^{\top}$ : sternite 5 usually with spinelike bristles along inner margins of lateral lobes; cerci usually curving anteriorly. 우: sternites $7-8$ of separate sclerites and form short ovipositor for larviposition; SPAP usualy absent............................... ...Tribe Sarcophagini …... 4

4. Gena entirely clothed with yellowish white hairs; $\mathrm{AS}_{3}$ entirely yellow orange (Tumrasvin and Kano, 1979: Fig. 14) ...

............ Liopygia Enderlein, L. ruficornis (Fabricius)

- Gena clothed with black hairs at least in part, largely on anterior part or entirely; $\mathrm{AS}_{3}$ largely or entirely fuscous black

5. Postsutural $a c$ absent or rarely present, but fine or weak, exceptionally strong in case of $R$. anchori-

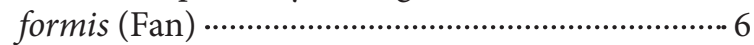

- Postsutural ac distinct, developed …………………. 8

6. Gena entirely clothed with black hairs; propleuron hairy; male stenite 5 with conical projection medially (Tumrasvin and Kano, 1979: Fig. 42) ..

.. Rosellea Rohdendorf,
R. khasiensis (Senior-White)

- Gena usually clothed with yellowish white hairs posteriorly, if entirely with black hairs, then, propleuron bare; male sternite 5 without conical median projection ………………………….................... 7

7. Male $\mathrm{GS}_{2}$ reddish; male hind femur without long fine hairs curled (Tumrasvin and Kano, 1979: Fig. 40) ……………….... Bercaea Robineau-Desvoidy, B. africa (Wiedemann)

- Male $\mathrm{GS}_{2}$ black; male hind femur with fine long hairs which are longer than width of femur and curled apically; female tergite 6 of two narrow sclerites . Robineauella Enderlein

8. Postsutural $d c 3$, each bristle subequal in length ..... Myorhina Robineau-Desvoidy

- Postsutural $d c 4$, each bristles subequal in length ... 9

-- Postsutural $d c 5$, sometimes 6-7, anterior 3-5 bristles less developed than hindmost two and gradually decreased in length toward transverse

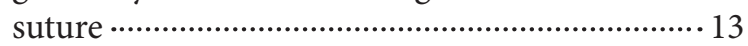

9. Propleuron bare ………………………………..... 11

- Propleuron hairy; tergite 6 in $0^{\gamma}$ divided into two narrow sclerites with $m b$..................................... 10

10. Yellowish white hairs on gena not extending forward to line drawing from posterior margin of eye; male sternite 4 usually with posterior pad of dense shorter clothing setae or upstanding long hairs, rarely without pad or upstanding long hairs in S. montana; aedeagus with slender stylus; pregonite bifurcate....... Sarcorohdendorfia Baranov

- Yellowish white hairs on gena extending forward to line drawing from posterior margin of eye; male sternite 4 without pad or upstanding long hairs posteriorly; adeagus with stout stylus; pregonite not branched ……………….... Lioproctia Enderlein

11. Medium sized flies, $8.5-10.0 \mathrm{~mm}$; body slender; hind tibia without fringe; row of presutural $a c$ developed in distinct row in $\sigma^{\gamma}$, single pair of presutural $a c$ distinct in P; tergite 8 of two lateral vestigial sclerites; sternite 8 membranous, with setulose hairs; alar squama with tuft of yellowish white hairs on inner lower margin ......... Sarcosolomonia Baranov/Fengia Rohdendorf - Large sized flies; body rather stout, more than $11 \mathrm{~mm}$; hind tibia with well developed fringe on both antero- and postero-ventoral surfaces; presutural $a c$ absent or single pair distinguishable just in front of suture; alar squama with tuft of fuscous black hairs on inner lower margin ……………..... 12

12. Male sternite 5 with small chitinous protuberance on middle part of ventral surface; gena clothed, 
intermixed with yellow hairs posteriorly (Tumrasvin and Kano, 1979: Fig. 44).......

.. Phallospaera Rohdendrof

- Male sternite 5 without such small chitinous protuberance on middle of ventral surface; gena clothed with black hairs only .... Burmanomyia Fan

13. Vein $\mathrm{R}_{1}$ setulose (Tumrasvin and Kano, 1979: Fig. 47) Alisarcophaga Fan \& Chen, A. gressitti (Hall \& Bohart)

- Vein $\mathrm{R}_{1}$ bare 14

14. Propleuron bare 16

- Propleuron hairy .15

15. Scutellum without discal scutellar bristle $(d s c)$; male sternite 4 without remarkable hairs on median part of posterior margin; ventralia not globose, without seration; hind tibia with well developed fringe on posteoventral surface (Tumrasvin and Kano, 1979: Fig. 1) .

.. Hosarcophaga Shinonaga \& Tumrasvin, H. serrata $(\mathrm{Ho})$

- Scutellum with one pair of $d s c$; male sternite 4 with rather long hairs on median part of posterior margin; ventralia large, globose, with seration and numerous minute spines; hind tibia without developed fringe on posteroventral surface in $\sigma^{\top}$, rarely with poorly developed fringe in part

.... Boettcherisca Rohdendorf/Rosellea Rohdendorf

16. Mid tibia without fringe in $\sigma^{7}$; ; tergite 6 incomplete, divided into two lateral narrow sclerites with mb ….................................................................. 17

- Mid tibia with fringe more or less developed in $0^{7}$; female tergite 6 developed, of complete plate, with row of strong marginal bristles ..

17. Vein $\mathrm{R}_{1}$ with distinct row of black setulae on basal $1 / 2$ of dorsal surface; costal spine rather long, longer than $2 \mathrm{X}$ width of costa; 우: sternite 8 present; tergite 8 of two small vestigial sclerites (Tumrasvin and Kano, 1979: Fig. 33) ....

........ Asceloctella Enderlein, A. calicifera (Böttcher)

- Vein $\mathrm{R}_{1}$ without setulae on dorsal surface; costal spine short, often not distinctly developed; 우: sternite 8 usually absent; tergite 8 of single or two narrow sclerites ……... Parasarcophaga Johnston \& Tiegs/Sinonipponia Rohdendorf

18. Gena largely clothed with black hairs anteriorly; sternite 4 densely haired posteriorly in $0^{7}$.............19

- Gena largely clothed with yellowish white hairs; sternite 4 without mat of hairs, but entirely with fine long hairs in $\sigma^{\top}$ (Nandi, 2002: Figs. 886-890) .... Iranihindia Rohdendorf,

*I. martellatoides (Baranov)
19. Mid tibia with well developed fringe on entire length and 2 ad medially in $\sigma^{7}$; hind tibia usually with well developed fringe, but without $a v$ in $\sigma^{\gamma}$, tergite 6 in o prominent in lateral view, of complete sclerite without median cleft (Tumrasvin and Kano, 1979: Fig. 46) ...... Seniorwhitea Rohdendorf,

S. princeps (Wiedemann)

- Mid tibia with poor fringe on apical $1 / 3$ and 1 submedian $a d$; hind tibia with poorly developed fringe on apical $2 / 3$ and 1 strong $a v$ in $\sigma^{\top}$ (Tumrasvin and Kano, 1979: Fig. 45)

Harpagophala Rohdendorf, H. kempi (Senior-White)

\section{Tribe Protodexiini}

\section{Key to the species of Blaesoxipha}

1. Tergite 3 with strong median $m b$; st $2+1 \ldots \ldots \ldots \ldots . . .2$

- Tergite 3 without median $m b ; s t 1+1$.................... 3

2. Palpus orange to dark brown; $\mathrm{AS}_{2}$ orange (Tumrasvin and Kano, 1979: Fig. 30) ............ B. pachytyli (Skuse)

- Palpus and $\mathrm{AS}_{2}$ blackish (Tumrasvin and Kano, 1979: Fig. 34) ………………. B. rufipes (Macquart)

3. Cercus in $\sigma^{\top}$ bent backward medially; aedeagus with spinose vesica; pregonite enlarged, curved upright (Tumrasvin and Kano, 1979: Fig. 32) .......... ................... B. thailandica Shinonaga \& Tumrasvin

- Cercus in $\sigma^{\top}$ not bent backward medially; aedeagus without spinose vesica; pregonite slender (Tumrasvin and Kano, 1979: Fig. 31)

B. unicolor Villeneuve

\section{Tribe Sarcophagini}

The postsutural $d c 3$ group

\section{Key to the species of Myorhina}

1. Cell $\mathrm{R}_{5}$ closed at wing margin; fore tibia with $2 p$; $\mathrm{CS}_{5}$ setulose along anterior margin on basal $1 / 2 \ldots .$. * M. otiophalla (Fan \& Chen)

- Cell $\mathrm{R}_{5}$ open; fore tibia with $1 p$; $\mathrm{CS}_{5}$ setulose along anterior margin almost entirely

2. Upper and lower lobes of ventralia projecting forward in parallel direction in lateral view. (Tumrasvin and Kano, 1979: Fig. 41).

M. caudagalli (Böttcher)

- Upper and lower lobes projecting to different direction, directed at right angle in lateral view [No record from Thailand] ………..... M. crinitula (Quo) 
The postsutural $d c 4$ group

\section{Key to the species of Sarcosolomonia/Fengia}

1. Tergite 3 with median $m b$; cercus with subapical spines in $\sigma^{7}$; mid tibia with $1 a$ (Tumrasvin and Kano, 1979: Fig. 29).

... S. shinonagai Kano \& Sooksri

- Tergite 3 without median $m b$; cercus in $\sigma^{7}$ without subapical spines . .2

2. Vein $\mathrm{R}_{1}$ setulose; gena clothed with yellow hairs posteriorly; 우 tergite 8 present, of two narrow lateral sclerites; 우 sternite 8 membranous, setulose; 우 sternite 5 present, triangular-shaped .................. 3

- Vein $R_{1}$ bare; gena entirely clothed with black hairs

3. Pregonite with flange; ventralia bifid at apex in $\sigma^{7}$ (Tumrasvin and Kano, 1979: Fig. 24)

S. rohdendorfi Nandi

- Pregonite without flange; ventralia pointed at apex (Tumrasvin and Kano, 1979: Fig. 27)

. S. trifulcata Shinonaga \& Tumrasvin

4. Gena entirely clothed with black hairs; hind tibia fringe on apical $2 / 3$ of anteroventral and posteroventral surfaces, sometimes not typically fringed, only with 4-5 fine long hairs on anteroventral surface, with $1 a v$; thoracic squama fuscous on disc (Tumrasvin and Kano, 1979: Fig. 38) F. ostindicae (Senior-White)

- Gena with yellow hairs posteriorly; hind tibia without fringe on posteroventral surface, with 2 $a v$; thoracic squama entirely pale brown …............ 5

5. Middle of anterior part of cercus with free part bent upward; cercus pointed at apex; juxta small in $\sigma^{7}$ (Tumrasvin and Kano, 1979: Fig. 26) .....

............ S. aureomarginata Shinonaga \& Tumrasvin

- Middle of anterior part of cercus normal, without free part bent upward; cercus forked at apex; juxta or harpe large (Tumrasvin and Kano, 1979: Fig. 25) ……................................. S. crinita (Parker)

\section{Key to the species of Burmanomyia ( $\left.\sigma^{7} \sigma^{7}\right)$}

1. Gena exclusively with black hairs; mid tibia without fringe...... .... 2

- Gena largely clothed with white hairs; mid tibia with well developed fringe

2. Ventralia chitinous, trilobate; juxta with cupshaped grooved apex and two projections; pregonite with pointed apex; cercus narrow, curved, without row of setulae (Nandi, 2002: Figs. 426430)

B. beesoni (Senior-White)

- Ventralia membranous, bilobate; juxta without groove and blind; pregonite broad medially, with row of about 8 setulae; cercus stout (Pape and Bänziger, 2003: Figs. 7-10)

B. suthep (Pape \& Bänziger)

3. Sternal lobes of sternite 5 much divergent, Vshaped, with open sternal window; cercus pointed apically; juxta rounded, serrated with 4 distinct teeth along anterolateral margin, with membranous appearance (Chaiwong et al., 2009: Fig. 3A) .. B. nanensis (Chaiwong, Sukontason \& Sukontason), n. comb.

- Sternal lobes of sternite 5 less divergent, Y-shaped, with closed sternal window; cercus with notch; juxta rounded, serrated with 1-4 distinct teeth along anterolateral margin, but without membranous appearance (Chaiwong et al., 2009: Fig. 3B) ..B. spinipenis (Shinonaga \& Tumrasvin), n. comb.

\section{Key to the species of Phallosphaera}

1. Three rows of black postocular setae regular; $\mathrm{AS}_{3}$ $3 \mathrm{X}$ as long as $\mathrm{AS}_{2}$; cercus large widely broadened on dorsal surface and curved at apical $1 / 2$, without tuft of long hairs near apex (Tumrasvin and Kano, 1979: Fig. 39)...

.................... P. kurahashii Shinonaga \& Tumrasvin

- Only one row of black postocular setae regular; $\mathrm{AS}_{3} 4 \mathrm{X}$ as long as $\mathrm{AS}_{2}$; cercus in $\sigma^{7}$ not so broad, stretched, with tuft of long hairs subapically (Tumrasvin and Kano, 1979: Fig. 44)

P. gravelyi (Senior-White)

\section{Key to the species of Lioproctia}

1. Abdomen with yellowish golden pollinosity on tergites 4-5 (Pape and Bänziger, 2000: Figs. 1-3) ... ………………... L. saprianovae (Pape \& Bänziger)

- Abdomen with ordinally greyish pollinosity on tergites 4-5 (Tumrasvin and Kano, 1979: Fig. 36) .. .. L. pattoni (Senior-White)

\section{Key to the species of Sarcorohdendorfia}

1. Wing yellowish orange basally (Tumrasvin and Kano, 1979: Fig. 22) ................. S. seniorwhitei (Ho)

- Wing hyaline …........................................................ 2

2. Male sternite 4 with patch of spine-like bristles postriorly ............................................................ 3

- Male sternite 4 without such patch ……….............. 5

3. Parafacial densely golden pollinose; epandrium/ male genital segment $2\left(\mathrm{GS}_{2}\right)$ black shining; hairs on sternite 4 long, equal in length to those of sternite 3; inner ridge of posterior surface of cercus with some fine long hairs and outer ridge with spines; ventralia two-wave-like shaped and its apical portion hook-like (Tumrasvin and Kano, 1979: Fig. 23) 
S. multivillosa (Shinonaga \& Tumrasvin)

- Parafacial silvery grey pollinose, newly with yellowish grey tinge; $\mathrm{GS}_{2}$ reddish orange; hairs on sternite 4 much shorter than those of sternite 3; inner ridge of posterior surface of cercus bare, only with spines at outer ridge; ventralia well developed

4. Ventralia serrated on apical $1 / 2$; cercus without spines on anterobasal corner (Tumrasvin and Kano, 1979: Fig. 15) ……..... S. inextricata (Walker)

- Ventralia without serration; cercus with 1-3 spines on anterobasal corner; + + tergite 6 and sternite 7 orange (Tumrasvin and Kano, 1979: Fig. 18) ............ S. antilope (Böttcher)

5. Mid tibia without $v$; gena largely clothed with yellowish hairs except for a few black ones on anterior extremity (Tumrasvin and Kano, 1979: Fig. 21) S. montana (Shinonaga \& Tumrasvin)

- Mid tibia with $v$; gena largely clothed with black hairs anteriorly (Tumrasvin and Kano, 1979: Fig. 36) - Lioproctia pattoni (Senior-White)

The postsutural $d c 5$ group

\section{Key to the species of Boettcherisca}

1. Gena clothed with black hairs only ... 2

- Gena more or less with yellowish hairs posteriorly

2. Thoracic squama fuscous on disc; alar squama with tuft of fuscous hairs on inner lower margin; $\mathrm{GS}_{2}$ blackish (Kurahashi and Tan, 2009: Fig. 2) ...... B. highlandica Kurahashi \& Tan

- Thoracic squama pale, white on disc; alar squama with tuft of yellowish white hairs on inner lower margin; $\mathrm{GS}_{2}$ reddish (Tumrasvin and Kano, 1979: Fig. 17) B. javanica Lopes

3. Abdomen golden pollinose on tergites 3-5 (Pape and Bänziger, 2000: Figs. 4-5) ...

$$
\text { ………………..... B. krathonmai (Pape \& Bänziger) }
$$

- Abdomen greyish pollinose on tergites 3-5 ……... 4

4. Yellow genal hairs extending forward to level of line drawing from posterior margin of eye; juxta of aedeagus with rounded apex; pregonite elongate, slender; spines on apical part of cercus not extending to dorsal surface (Tumrasvin and Kano, 1979: Fig. 19) … B. peregrina (Robineau-Desvoidy)

- Yellow hairs not extending forward to level of line projecting from posterior margin of eye; juxta two-lobulated apically; pregonite rather stout; spines on apical part of cercus extending to dorsal surface (Tumrasvin and Kano, 1979: Fig. 20) ...........

\section{Key to the species of Robineauella $\left(\sigma^{7} \sigma^{7}\right)$}

1. Postsutural ac developed as prescutellar one ............ * R. anchoriformis (Fan)

- Postsutural $a c$ absent, fine or weak ………………... 2

2. Gena with yellowish white hairs posteiorly . R. thailandica sp. nov.

- Gena clothed with black hairs only ……………….... 3

3. Mid tibia with 2 ad (Rohdendorf, 1966: Figs. 1-2).. . R. coei Rohdendorf

- Mid tibia with 1 ad (Fan, 1965: Figs. 1116-1117) ... *R. pseudoscoparia (Kramer)

\section{Key to the species of Parasarcophaga $\left(\sigma^{7} \sigma^{7}\right)$}

1. Palpus at least yellowish orange apically ………….. 2

- Palpus fuscous brown to black ……………………… 3

2. Palpus entirely yellowish orange; propleuron bare (Tumrasvin and Kano, 1979: Fig. 4)

. P. (Parasarcophaga) misera (Walker)

- Palpus yellowish, darkened on basl 1/3; propleuron sometimes hairy (Kurahashi and Sukontason, 2004: Fig. 1).

.......... Sinonipponia komi Kurahashi \& Sukontason

3. Sternite 4 conspicuously haired; mid femur without comb-like posteroventral bristles; hind tibia never fringed; stylus large, covered with minute hairs (Tumrasvin and Kano, 1979: Fig. 2)

P. (Kanomyia) bangkokensis Shinonaga \& Tumrasvin

- Sternite 4 sparsely haired; mid femur with comblike posteroventral bristles on apical part; hind tibia usually fringed; stylus slender, never covered with minute hairs

. .4

4. Ventralia pedunculated

- Ventralia not pedunculated ....................................... 7

5. One row of postocular setae present; gena with only a few black hairs at foremost anterior part; hind femur without fringe; ac $1+1$ (Tumrasvin and Kano, 1979: Fig. 3)

........ P. (Parasarcophaga) taenionota (Wiedemann)

- Three rows of black postocular setae present, only first row regular; gena with numerous black hairs anteriorly; hind femur with fringe; ac $0-1+1 \ldots . .6$

6. Hind tibia with 1 av (Tumrasvin and Kano, 1979: Fig. 5) ……. P. (Parasarcophaga) albiceps (Meigen)

- Hind tibia without av (Rohdendorf, 1937: Figs. 274-275)

............. P. (Parasarcophaga) hirtipes (Wiedemann)

7. Sternites $2-3$ with dense long hairs, length of 
which longer than that of each sternite (Tumrasvin and Kano, 1979: Fig. 6)

$$
\text { P. (Liosarcophaga) javana (Macquart) }
$$

- Hairs on sternites 2-3 shorter than length of each sternite

8. Hind tibia without fringe ………………………..... 9

- Hind tibia with fringe ……………………………... 12

9. Juxta small, with lateral processes, bifid at apex (Tumrasvin and Kano, 1979: Fig. 8) .....

...P. (Liosarcophaga) scopariiformis (Senior-White)

- Juxta elongated, with median apophysis ............... 10

10. Mid tibia with 2 ad; membranous lobe of juxta rod-like, blunt at apex (Fan, 1965, Figs. 11081110) …………………. P. (Curranea) iwuensis Ho

- Mid tibia with $1 \mathrm{ad}$; membranous lobe of juxta divergent and curled at apex

11. Wing infuscated especially along veins; ventralia beak-shape apically (Fan, 1965, Figs. 1104-1105) .. *P. (Curranea) hinglungensis Fan

- Wing hyaline; ventralia with ring-like head (Fan, 1965: Figs. 1106-1107) P. (Curranea) yunnanensis Fan

12. Only 1 row of black postocular setae present …. 13

- More than one row of black postocular setae present

13. Cercus enlarged dorsally and with small spines along anterior margin of dorsal surface; ventralia composed of 2 lobes, lower one serrated; lateral arm of juxta pointed at apex (Tumrasvin and Kano, 1979: Fig. 9) ………. P. (Liosarcophaga) amplicercus Shinonaga \& Tumrasvin

- Cercus normal in shape, not enlarged dorsally and without spines; ventralia composed of 1 lobe; lateral arm of juxta bifid at apex (Tumrasvin and Kano, 1979: Fig. 13)

$$
\text { P. (Liosarcophaga) dux (Thomson) }
$$

14. Black genal hairs located only in anteior part of gena; cercus beak-shape at apex (Tumrasvin and Kano, 1979: Fig. 11)

... P. (Liosarcophaga) brevicornis $(\mathrm{Ho})$

- Black genal hairs extending to postgena beyond anterior $1 / 2$ of gena; cercus pointed at apex (Tumrasvin and Kano, 1979: Fig. 16)

P. (Liosarcophaga) idmais (Séguy)

New Taxon

\section{Robineauella thailandica sp. nov.}

DESCRIPTION. $\sigma^{\top}$. - Head: dichoptic; frons index $0.20-0.21(\mathrm{M}=0.20, \mathrm{n}=4)$; frontal stripe black; para- facial grey pollinose; antenna blackish, slightly grey pollinose, 3rd segment $\left(\mathrm{AS}_{3}\right) 2.5 \times$ as long as $2 \mathrm{nd}\left(\mathrm{AS}_{2}\right)$; gena black, grey pollinose, with numeous black hairs except for several yellowish white hairs posteriorly, ca. $1 / 4$ of height of eye (HE); postgena with numerous yellowish white hairs; ori 11-12, lower 3-4 divergent; 1st and 2nd rows of postocular/postorbital setae regularly arranged, but others irregular in row. Palpus black, slender, with black bristles and hairs.

Thorax: black, grey pollinose, with 3 black longitudinal stripes on scutum; propleuron bare; prosternum hairy on posterior $2 / 3$; mesothorcic and metathoracic spiracles blackish. Chaetotaxy: $a c 0+0$; $d c 5+5-6$; ia $1+1-4$, hindmost one well developed; $h$; ph 1 ; prs 1 ; sa 3 ; $n$; pa 2 ; sc $4+1 ; s t 1+1+1$.

Wings: hyaline; epaulet black; basicosta creamy white; vein $R_{1}$ bare; vein $R_{4+5}$ with row of 9-12 short setulae extending $1 / 2$ way from basal node to $\mathrm{r}-\mathrm{m}$ above; several setulae on node of veins $R_{2+3}$ and $R_{3}+5$ below; $\mathrm{CS}_{3}$ longer than $\mathrm{CS}_{5} ; \mathrm{CS}_{5}$ with short spines along anterior margin on basal 2/5. Alar and thorcic squamae both pale brown, alar squama with tuft of fuscous brown hairs on inner lower margin. Halter blackish, fuscous brown on basal 1/2.

Legs: black; fore femur with 2 rows of long $p d$, row of long $p v$; fore tibia with 3 short ad basally, $1 p$ at apical $1 / 3$; mid femur with numerous long apically curled hairs on slightly longer than basal $1 / 2$ of posteroventral surface, 4 strong $a$ present along median longitudinal line, $2 p$-pd apically, row of rather strong $a v$, rows of short and stout $p v$ apically; mid tibia with 2 submedian $a d, 1$ $p d, 1 p$ and $1 v$ present at apical $1 / 3$; hind femur with 2 rows of $a d$ on basal $1 / 2$, row of $a v$, and $2 p$-pd apically, numerous long and apically curled hairs present on antero- and posteroventral surfaces; hind tibia with $2 \mathrm{ad}, 2$ $p d$, and 1 av at apical $1 / 3$, with well developed fringes on anteroventral and posteroventral surfaces.

Abdomen: black, grey to whitish grey pollinose, tessellate; tergite 3 without marginal bristle $(\mathrm{mb})$; tergite 4 with pairs of median and 2 lateral marginal bristles; tergite 5 with row of strong marginal bristles; sternite 5 of Y-shape; $\mathrm{GS}_{1}$ fuscous black, more or less covered with brownish-grey pollinosity, with several fine bristly hairs; epandrium $\left(\mathrm{GS}_{2}\right)$ black shining, with numerous black bristly hairs. Hypopygium: as shown in Figs. 1-2.

ㅇ. - Unknown.

Length: $10.0-11.0 \mathrm{~mm}$.

Type meterial. Holotype: $\sigma^{7}$, "Holotype [red card]// THAILAND: CHIANG MAI/Doi Nang Kaew/Doi Saked/972 m, 7.III.2007/coll. Tarinee Chaiwong//Robineuella $\sigma^{7} /$ thailandica/sp. nov./det. H. Kurahashi [red perimeter]". Paratypes: with "Paratype [yellow card] and identification label [blue perimeter]//Robineuella $\sigma^{7} /$ thailandica/sp. nov./det. H. Kurhashi" THAILAND: CHIANG MAI: $1 \sigma^{\nearrow}$, Mae Rim Dist. T. Mae Raem, Tardmok waterfall, 27.V.2009, Coll. K. Moophayak; $10^{7}$, Muang, Doi Suthep-Pui, N18.47'19"E 98.55'15", 
918 m, 13.xi.2009, Coll. Kittikhun Moophayak; $10^{7}$, Muang, Doi Suthep-Pui, 950 m, 98.54'34"N, 18.48'20" E, 20.IX.2010, Coll. R. Ngoen-klan, three paratype specimens with a small tube containing dissected genitalia.

Etymology. The specific epithet is originated from the name of country "Thailand".

Type depository. Holotype (type series NSMT-IDip6841) and one paratype (type series NSMT-IDip6842) are deposited in National Museum of Nature and Science, Tsukuba. One paratype separately in Department of Parasitology, Faculty of Medicine, Chiang Mai Univesity, Chiang Mai (DPCM) and Bishop Museum, Honolulu (BPBM).

Remarks. The present new species is similar to Robineauella coei Rohdendorf, 1966 from Nepal and the widespread distributed species $R$. pseudoscoparia (Kramer, 1911) in general appearance, but easily distinguished from these two previously known species by the characteristic shape of male genitalia having the truncate apex of juxta in lateral view (Fig. 2b) and cercus without spinous setulae along anteior margin (Fig. 2c).

Bionomics. Robineauella thailandica sp. nov. seems to be rare and indigenous in Thailand. Small numbers of male flies were collected along mountain path in well preserved natural forests around Chiang Mai, Thailand.

Distribution. Thailand.

\section{Check List}

The following is the species list of Thai Sarcophagidae mainly based upon specimens examined, but partly upon published records in the case of no available material.

\section{Subfamily MILTOGRAMMATINAE}

\section{Tribe Amobiini}

\section{*Amobia auriceps (Baranov, 1935)}

THAILAND: 2우, Khao Yai, $30 \mathrm{~km}$ S. Pak Chong, 25-26.xii.1975, S. Shinonaga (NSMT); 1우, Chiang Mai Prov., Doi Saket District, Doi Nang Kaew, 1,016 m, 10.iii.20112, H. Kurahashi (NSMT).

\section{*Amobia quatei Kurahashi, 1974}

THAILAND: 10 $\sigma^{7}$, Kanchana Buri, Erawan w. f., 500 m, 7.ix.1975, H. Kurahashi (NIID); 1우, Chiang Mai Prov., Doi Saket District, Doi Nang Kaew, 19.03'53"N, 99.22'34"E, 1,142 m, 10.iii.2011, K. Moophayak (DPCM).

\section{Tribe Miltogrammatini}

*Hoplacephala linearis Villeneuve, 1929 THAILAND: 1우, Chiang Mai Prov., Doi Suthep-Pui National Park, Headquarters area, 1,075 m, 4-5.iii.2007, H. Kurahashi (IDD) $[1$ 우>DPCM].

Miltogramma angustifrons (Townsend, 1933)

THAILAND: $20^{7}$, Kanchana Buri, nr. Sai Yok, 500 m, 8.x.1975, H.
Kurahashi (NIID); 1우, Chiang Mai Prov., Doi Inthanon, 1,300 m, A7, 10-12.v.1988, H. Kurahashi (NIID); 47ð', Chiang Mai Prov., Doi Saked, Ban Pangdoeng, 508 m, 25.vii.2007, H. Kurahashi (IDD) $\left[7 \sigma^{\top}>\mathrm{DPCM}\right.$ (gift), $7 \sigma^{\top}>\mathrm{BPBM}$ (gift), $7 \sigma^{\top}>\mathrm{BMNH}$ (gift), $7 \sigma^{\text {T }}>\mathrm{ZMUC}$ (gift), $70^{\text {乃 }}>\mathrm{CMNH}$ (gift)].

*Miltogramma iberica Villeneuve, 1912

THAILAND: 10', Fang, Doi Huai Hwer, 1,281 m, 27.ix.1975, H. Kurahashi (NIID); 10 $\sigma^{7}$, Kanchana Buri, nr. Sai Yok, 500 m, 8.x.1975, H. Kurahashi (NIID).

Protomiltogramma kabkaewae Kurahashi \& Chaiwong, 2007

THAILAND: 1우, Chiang Mai Prov., Mae Taeng Dist., Mae Taeng Elephant Camp, 345 m, 14.xi.2011, H. Kurahashi (IDD).

Protomiltogramma komi Kurahashi \& Chaiwong, 2007

THAILAND: Holotype $\sigma^{7}$, Chon Buri, c. $30 \mathrm{~km} \mathrm{~S}$, beach, 30 . viii.1975, R. Kano (NSMT).

Protomiltogramma macularis (Wiedemann, 1824) THAILAND: 1우, Bangkok, Mahidol Univ., det. by Pape as Miltogramma macularis, 14.ix.1975, H. Kurahashi (NSMT); 10', Chiang Mai Prov., Mae Taeng Dist., Mae Taeng Elephant Camp, 345 m, 14.xi.2011, T. Klongkiaew (DPCM); 1우, Chiang Mai Prov., Mae Taeng Dist., Mae Taeng Elephant Camp, 345 m, 14.xi.2011, H. Kurahashi (IDD).

\section{*Senotainia albifrons Rondani, 1859}

THAILAND: 1우, Chon Buri, c $30 \mathrm{~km} \mathrm{~S}$, beach, 30.viii.1975, H. Kurahashi (NIID).

\section{Senotainia navigatrix (Meigen, 1910)}

THAILAND: 10', Doi Inthanon, Ban Yang, 1,400 m, 19.ix.1975, R. Kano (NIID); $10^{7}$ 2우, Kanchana Buri, nr. Sai Yok, 500 m, 6, 9.ix.1975, H. Kurahashi (NIID); 10 , nr. Ban Sap Bon, 30 km E. Sara Buri, 4.x.1975, W. Tumrasvin (NIID); $10^{\top}$, Khao Yai, 30 km S.

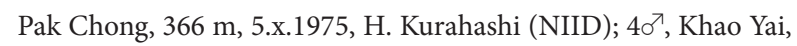
30 km S. Pak Chong, 25-26.xii.1975, S. Shinonaga (NIID); $5 \sigma^{7}$ 5우, Kanchana Buri, Sai Yok, 500 m, 27-29.xii.1975, S. Shinonaga and W. Tumrasvin (NIID); 10 , Chiangmai Prov., Doi Sthep, 1,000 m, 9-15.v.1988, H. Kurahashi (NIID); 2ð', Fang, Doi Huai Hwer, 1,231 m, 27.ix.1975, H. Kurahashi (NIID); 1우, Kanchana Buri, Sai Yok, 500 m, 9-13.xii.1975, S. Shinonaga (NIID); 10 , Nakon-nayok, 14.v.1962,? (NIID); 10', Bang Khen, 11.iv.1967,? (NIID); 1우, Fang, Doi Huai Hwer, 1,231 m, 27.ix.1975, R. Kano (NIID); $10^{7}$, Tak Prov., $80 \mathrm{~km} \mathrm{NW}$ of Mae Sot, Mae Rayang, 9.vi.1988, H. Kurahashi (NIID); $100^{\top} 2$ 우, Hill, 30 km SE Sa Kaeo, 300 m, 3.ix.1975, R. Kano and H. Kurahashi (NIID); 1우, Chiang Mai Prov., Doi Suthep-Pui National Park, Sirindhorn Observatory, 817 m, 4-5.iii.2007, H. Kurahashi (IDD) [1우>DPCM(gift)].

\section{Taxigramma multipunctata (Rondani, 1859)}

THAILAND: $30^{\top}$, Tak Prov., $65 \mathrm{~km}$ NE of Mae Sot, Ban Mai, 8.vi.1988, H. Kurahashi (NIID); 10 $\sigma^{\top}$, Chon Buri, c $30 \mathrm{~km} \mathrm{~S}$, beach, 30.viii.1975, R. Kano (NIID). 


\section{Tribe Metopiini}

*Metopia argentata Macquart, 1850

THAILAND: 1 우, Chiang Mai Prov., Mae Tang Dist., Mae Taeng Elephant Camp, 345 m, 14.xi.2011, H. Kurahashi (IDD).

\section{* Metopia argyrocephala (Meigen, 1824)}

THAILAND: $20^{\top}$, Kanchana Buri, nr. Sai Yok, 500 m, 11.ix.1975, H. Kurahashi (NIID); 30', Kanchana Buri, Sai Yok, 500 m, 9-13. xii.1975, 27-29.xii.1975, H. Shima (NIID); $10^{\top 7} 1$ 우, Doi Inthanon, Ban Yang, 1,400 m, 19.ix.1975, R. Kano (NIID); 10', Chiang Mai Prov., Maung, Huey Tueng Tao, lake, 9.iii.2007, H. Kurahashi (IDD) $\left[10^{\top}>\operatorname{DPCM}(\mathrm{gift})\right]$; $10^{\top}$, Chiang Mai Prov., Doi SuthepPui National Park, Sirindhorn Observatory, 817 m, 27.vii.2007, H. Kurahashi (IDD) $\left[10^{7}>\right.$ DPCM(gift)].

*Metopia nudibasis (Malloch, 1930)

THAILAND: 1우, Kanchana Buri, Sai Yok, 500 m, 27-29.xii.1975, W. Tumrasvin (NIID).

\section{* Metopia sauteri (Townsend, 1932)}

THAILAND: 1 우, Lampang Prov., Hang Chat Dist., Doi Khun Tan rest area, 523 m, 28.vii.2007, H. Kurahashi (IDD) [1우> $\operatorname{DPCM}($ gift)].

\section{Metopia suifenhoensis Fan, 1965}

THAILAND: $10^{\top} 1$ 우, Chiang Mai Prov., Doi Sthep, 1,000 m, 9-15.v.1988, H. Kurahashi (NIID); $10^{7}$, NW Chiangmai, Fang, 500 m, No. 357, 12-19.iv.1958, T. C. Maa (BРBM).

\section{Metopia tshernovae Rohdendorf, 1955}

THAILAND: $10^{7}$, Chiang Mai Prov., Doi Inthanon, Ban Yang, 1,400 m, Metopia tshernovae Rhd. det. by T. Pape, 1993, 17.ix.1975, H. Kurahashi (NSMT).

Metopia yunnanica Chao \& Zhang, 1988 THAILAND: $10^{7}$, Chiang Mai Prov., Chom Thong, Doi Inthanon, $1,494 \mathrm{~m}, 11 . x i .2009, \mathrm{H}$. Kurahashi (IDD) [1 $\left.0^{7}>\mathrm{UM}(\mathrm{exp})\right]$.

\section{Subfamily SARCOPHAGINAE}

\section{Tribe Protodexiini}

Blaesoxipha pachytyli (Skuse, 1891)

Blaesoxipha pachytyli: Tumrasvin \& Kano, 1979: 164.

\section{Blaesoxipha rufipes (Macquart, 1839)}

THAILAND: 1우, Ban Pong Din, $10 \mathrm{~km}$ NE. Doi Saket, 20. ix.1975, R. Kano (NIID); 10', Kanchana Buri, L[R]iver Kuwai, 18.vii.1978, H. Suzuki (NIID).

Blaesoxipha thailandica Shinonaga \& Tumrasvin, 1979

Blaesoxipha thailandica: Tumrasvin \& Kano, 1979: 165.

Blaesoxipha unicolor Villeneuve, 1912

Blaesoxipha unicolor: Tumrasvin \& Kano, 1979: 165.

\section{Tribe Sarcophagini}

Leucomyia alba (Schiner, 1868)

THAILAND: $10^{\top} 1$ 우, Surat Thani Prov., Ko Samui, Tong Yang, A3, 22.iv.1988, H. Kurahashi (NIID); $10^{7} 1$ 우, Chon Buri Prov., Pattaya, nr beach, A5, 5.v.1988, H. Kurahashi (NIID). SAMET IS.: 10', Rayon, 26.viii.1986, Y. Wada (NIID).

\section{The postsutural $d c 3$ group}

\section{Myorhina caudagalli (Böttcher, 1912)}

THAILAND: $10^{\top}$, Tak Prov., $65 \mathrm{~km}$ NE of Mae Sot, Ban Mai, 8.iv.1988, H. Kurahashi (NIID); $190^{\top}$, Chiang Mai Prov., Hang Dong Dist., T. Nongkaeo, 18.40'52"N 98.55'57"E, 360 m, 9. iii.2011, H. Kurahashi (IDD) [30 $>\mathrm{UM}(\exp )]$; $10^{\top}$, Chiang Mai Prov., Doi Suthep-Pui National Park, Sirindhorn Observatory, 817 m, 11.iii.2011, H. Kurahashi (IDD); 80 , Chiang Mai Prov., Ping river, San Sai, 9.iii.2007, H. Kurahashi (IDD) $\left[70^{\Upsilon 7}>\right.$ DPCM(gift)]; $10^{7}$, Chiang Mai Prov., Doi Suthep-Pui National Park, Sirindhorn Observatory, 817 m, 11.iii.2011, H. Kurahashi (IDD); $190^{\top}$, Chiang Mai Prov., Hang Dong Dist., T. Nongkaeo, $18.40^{\prime} 52^{\prime \prime} \mathrm{N} 98.55^{\prime} 57^{\prime \prime} \mathrm{E}, 360 \mathrm{~m}$, 9.iii.2011, H. Kurahashi (IDD) [3 $\left.\sigma^{\top}>\mathrm{UM}(\exp )\right]$.

Myorhina globovesica (Ye, 1980)

THAILAND: $10^{\top}$, Lampang Prov., Hang Chat Dist., Doi Khun Tan rest area, 523 m, 28.vii.2007, H. Kurahashi (IDD).

Myorhina lanna (Pape \& Bänziger, 2003)

Sarcophaga (Mehria) lanna Pape \& Bänziger, 2003: 52.

Myorhina melania Shinonaga \& Tumrasvin, 1979

Pierretia melania Shinonaga \& Tumrasvin, 1979: 139.

*Myorhina otiophalla (Fan \& Chen, 1981) THAILAND: 10', Kanchanabri, Sai Yok, 500 m, 27-29.xii.1975, S. Shinonaga (IDD).

The postsutural $d c 4$ group

Burmanomyia beesoni (Senior-White, 1924)

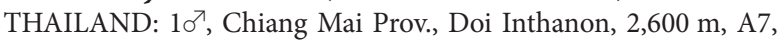
10-12.v.1988, H. Kurahashi (NIID); 107, Chiang Mai Prov., Doi Sthep, 1,000 m, A6, 9-15.v.1988, H. Kurahashi (NIID); 70', Chiang Mai Prov., Doi Inthanon, 1,300 m, A7, 10-12.v.1988, H. Kurahashi (NIID).

Burmanomyia nanensis (Chaiwong, Sukontason \& Sukontason, 2009), n. comb.

THAILAND: $2 \sigma^{7}$, Chiang Rai Prov., Maung, HueyTueng Tao, lake side, 3.xii.2007, H. Kurahashi (IDD).

Burmanomyia spinipenis (Shinonaga \& Tumrasvin, 1979), n. comb.

=Sarcophaga kiyokoae Chaiwong, Sukontason \& Sukontason, 2009, n. syn.

Burmanomyia suthep (Pape \& Bänziger, 2003)

THAILAND: $10^{\gamma}$, Chiang Mai Prov., Doi Suthep-Pui National 
Park, Sirindhorn Observatory, 817 m, 4-5.iii.2007, H. Kurahashi (IDD); $10^{7}$, Chiang Mai Prov., Muang, Doi Suthep-Pui Mount, 918 m, 18.47'19"N, 98.55'15"E, 13.xi.2009, H. Kurahashi (IDD); $10^{\top}$, Chiang Mai Prov., Muang, Doi Suthep-Pui Mount, Tham Phra Leusri, 950 m, 11.iii.2011, K. Moophayak (IDD).

\section{Fengia ostindicae (Senior-White, 1924)}

THAILAND: $10^{\top}$, Khao Yai, 10.iv.1963,? (NIID); 10', Chiang Mai Prov., Doi Saket, Doi, Nang Kaew, 1,010 m, 25.vii.2007, N. Bunchu (NSMT); $10^{\top}$, Chiang Mai Prov., Doi Saket, Doi Nang Kaew, 1,016 m, 10.iii.2011, H. Kurahashi (IDD); 10' Chiang Mai Prov., Doi Suthep-Pui National Park, Sirindhorn Observatory, 817 m, 11.iii.2011, H. Kurahashi (IDD); 10', Chiang Mai Prov., Doi Saket, Doi, Nang Kaew, 1,010 m, 25.vii.2007, N. Bunchu (NSMT); $10^{7}$, Chiang Mai Prov., Doi Saked-Ban Pang Deang, Nam Mae Kuang Bridge, 551 m, 7-8.iii.2007, H. Kurahashi (IDD); $30^{\top}$, Chiang Rai Prov., Doi Suthep-Pui National Park, Sirindhorn Observatory, 817 m, 7.xii.2007, H. Kurahashi (IDD); 607' Chiang Rai Prov., Pa Tuena Dist., Mae Chan, Huai Mahin Fon fall, 500 m, 5.xii.2007, H. Kurahashi (IDD) [60 $>$ DPCM(gift)]; $1 \sigma^{\top}$, Chiang Mai Prov., Doi Suthep-Pui National Park, Sirindhorn Observatory, 817 m, 27.vii.2007, H. Kurahashi (IDD); 20', Lampang Prov., Hang Chat Dist., Doi Khun Tan rest area, $523 \mathrm{~m}$, 28.vii.2007, H. Kurahashi (IDD); $2 \sigma^{\top}$, Lampang Prov., Hang Chat Dist., Doi Khun Tan, 523 m, 8.xii.2007, H. Kurahashi (IDD) [20 $>\operatorname{DPCM}$ (gift)]; $2 \sigma^{\top} 1$ 우, Chiang Mai Prov., Muang, Doi SuthepPui, 918 m, 13.xi.2009, H. Kurahashi (IDD) $\left[10^{\top}>\mathrm{UM}(\exp ), 1 \sigma^{\Upsilon}\right.$ 1 우>NSMT(gift)]; 10', Chiang Rai Prov., Hang Dong Dist., Samoeng-Hang Dong rd., 390 m, 9.xii.2007, H. Kurahashi (IDD) $\left[10^{\top}>\operatorname{DPCM}(\mathrm{gift})\right] ; 1 \sigma^{\Upsilon}$, Chiang Mai Prov., Hang Dong Dist., Huay Mae Khanin, 552 m, 13.iii.2007, H. Kurahashi (IDD); 10 Chiang Mai Prov., Doi Suthep-Pui National Park, Sirindhorn Observatory, 817 m, 11.iii.2011, H. Kurahashi (IDD); 10 , Chiang Mai Prov., Doi Saket, Doi Nang Kaew, 1,016 m, 10.iii.2011, H. Kurahashi (IDD); $10^{\top}$, Chiang Mai Prov., Doi Suthep-Pui Mt., Siridhrn Observatory, 817 m, 15.xi.2011, H. Kurahashi (IDD); $10^{7}$, Chiang Mai Prov., Muang, Doi Suthep-Pui Mount, Tham Phra Leusri, $950 \mathrm{~m}, 11$. iii.2011, H. Kurahashi (IDD) $\left[10^{\top}>\right.$ NSMT(gift)].

\section{Lioproctia pattoni (Senior-White, 1924)}

THAILAND: $10^{7}$, Surat Thani Prov., Ko Samui, N. Hinlad, A2, 20-21.iv.1988, H. Kurahashi (NIID); $30^{\top}$, Chiang Mai Prov., Zoo Chiang Mai, 10.iii.2003, K. Sukontason (DPCM); 10 , Chiangmai Prov., Chaingmai, 9.ix.1989, H. Kurahashi (NIID); 10 , Chiang Mai Prov., Doi Inthanon, 1,300 m, A7, 10-12.v.1988, H. Kurahashi (NIID); $1 \sigma^{\top}$, Chiang Mai Prov., Doi Saked, Doi Nang Kaew, $1,142 \mathrm{~m}, 19.03^{\prime} 53^{\prime \prime} \mathrm{N}, 99.22^{\prime} 34^{\prime \prime} \mathrm{E}, 14 . x i .2009$, T. Klongkiaew (IDD); $3 \sigma^{\nearrow}$, Chiang Mai Prov., Hang Dong Dist., Hana Dona,

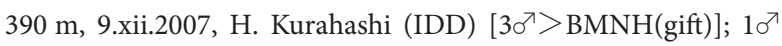
3 우, Chiang Mai Prov., Doi Suthep-Pui National Park, Sirindhorn Observatory, 817 m, 27.vii.2007, H. Kurahashi (IDD) [10 3우> $\operatorname{DPCM}$ (gift)]; $30^{7} 1$ 우, Chiang Mai Prov., Doi Suthep-Pui National Park, Sirindhorn Observatory, 817 m, 7.xii.2007, H. Kurahashi (IDD) $\left[30^{7} 1\right.$ 우 $>\mathrm{DPCM}$ (gift)]; $3 \sigma^{\urcorner}$, Chiang Mai Prov., Doi SakedBan Pang Deang, Nam Mae Kuang Bridge, 551 m, 7-8.iii.2007, H.

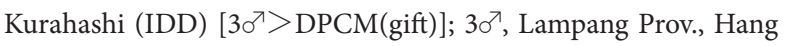

Chat Dist., Doi Khun Tan, 523 m, 8.xii.2007, H. Kurahashi (IDD); $10^{7}$, Chiang Mai Prov., Doi Suthep-Pui, 918 m, 18.47'19"N, 98.55' 15 "E,13.xi.2009, H. Kurahashi (IDD); 1우, Chiang Mai Prov., Doi Saked, Doi Nang Kaew, 1,010 m, 26.vii.2007, H. Kurahashi (IDD); $20^{\top}$, Chiang Mai Prov., Hang Dong Dist., Samoeng-Hang Dong rd., 390 m, 9.xii.2007, H. Kurahashi (IDD); 20 ${ }^{\top}$, Lampang Prov., Hang Chat Dist., Doi Khun Tan rest area, 523 m, 28.vii.2007, H. Kurahashi (IDD); $70^{7}$, Lampang Prov., Hang Chat Dist., Doi Khun Tan, 523 m, 8.xii.2007, H. Kurahashi (IDD) $\left[70^{\top}>\right.$ BPBM(gift)]; $10^{\top}$, Chiang Mai Prov., Maung, HueyTueng Tao, 3.

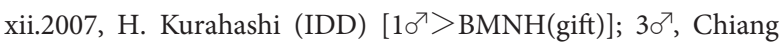
Mai Prov., Muang, Doi Suthep-Pui, 918 m, 18.47'19"N, 98.55'15" E, 13.xi.2009, H. Kurahashi (IDD) $\left[20^{\top}>\mathrm{UM}(\exp ), 10^{\top}>\right.$ NSMT(gift)]; 3우, Chiang Mai Prov., Doi Saket, Doi Nang Kaew, 1,142 m, 19.03'53"N, 99.22'34"E, 14.xi.2009, H. Kurahashi (IDD) [2우> UM(exp), 1우>NSMT(gift)]; $5 \sigma^{\nearrow}$, Chiang Mai Prov., Doi Suthep-Pui National Park, Sirindhorn Observatory, 817 m, 11.iii.2011, H. Kurahashi (IDD) [20 $\left.70^{7}>\mathrm{UM}(\exp )\right]$; 1우, Chiang Mai Prov., Doi Suthep-Pui Mt., Siridhrn Observatory, 817 m, 15.xi.2011, H. Kurahashi (IDD); $20^{\top} 3$ 우, Chiang Mai Prov., Mae Taeng Dist., Mae Taeng Elephant Camp, 345 m, 14.xi.2011, H. Kurahashi (IDD); $50^{\top}$, Chiang Mai Prov., Doi Suthep-Pui National Park, Sirindhorn Observatory, 817 m, 11.iii.2011, H. Kurahashi (IDD) $\left[2 \sigma^{\top}>\mathrm{UM}(\exp )\right]$.

Lioproctia saprianovae (Pape \& Bänziger, 2000)

THAILAND: $10^{7} \mathrm{PT}$, Tak Prov., Umphang Distr., Ban Thi Pho Ji, path to Büng La Ü Ö, 810 m, 7.ii.1999, H. Bänziger (SMNH) [107 PT>NIID]; $10^{\top}$, Chiang Mai Prov., Doi Saked, Doi Nang Kaew, $1,142 \mathrm{~m}, 19.03^{\prime} 53^{\prime \prime} \mathrm{N}, 99.22^{\prime} 34^{\prime \prime} \mathrm{E}, 14 . x i .2009$, T. Klongkiaew (IDD); $110^{\gamma} 5$ 우, Chiang Mai Prov., Doi Saket, Doi Nang Kaew, 13.xi.2011, H. Kurahashi (IDD) $\left[20^{\top} 1\right.$ 우 $>\mathrm{DPCM}$ (gift); $20^{\top}>$ $\mathrm{UM}(\exp )]$.

Phallosphaera gravelyi (Senior-White, 1924)

THAILAND: $10^{7}$, Chiang Mai Prov., Doi Saket, Doi Nang Kaew, 1,016 m, 10.iii.2011, H. Kurahashi (IDD).

\section{Phallosphaera kurahashii Shinonaga \& Tumrasvin,} 1979

THAIlAND: $10^{\top}$, Chiang Mai Prov., Doi Sthep, 1,000 m, 9-15. v.1988, H. Kurahashi (NIID).

\section{Sarcorohdendorfia antilope (Böttcher, 1913)}

THAILAND: $2 \sigma^{\top}$, Chiang Mai Prov., Doi Inthanon, 1,300 m, A7, 10-12.v.1988, H. Kurahashi (NIID); $140^{\nearrow}$, Chiang Mai Prov., Doi Saket, Doi Nang Kaew, 1,016 m, 10.iii.2011, H. Kurahashi (IDD)

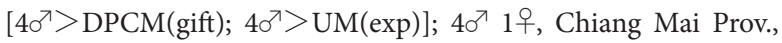
Doi Suthep-Pui National Park, Sirindhorn Observatory, 817 m, 11.iii.2011, H. Kurahashi (IDD); 3o', Chiang Mai Prov., Muang, Doi Suthep-Pui Mount, Tham Phra Leusri, 950 m, 11.iii.2011, H. Kurahashi (IDD); $1 \sigma^{7}$, Chiang Mai Prov., Doi Inthanon, 1,300 m, A7, 10-12.v.1988, H. Kurahashi (IDD) [10 $>$ NSMT(gift)]; $10^{7}$, Chiang Mai Prov., Doi Saket, Ban Pang Deang, Nam Mae Kuang Bridge, $551 \mathrm{~m}, 7-8$. iii.2007, H. Kurahashi (IDD) $\left[10^{\top}>\right.$ NSMT(gift)]; $1 \sigma^{\top}$, Chiang Mai Prov., Doi Saket, Doi Nang Kaew,

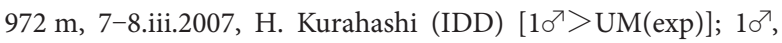


Lampang Prov., Hang Chat Dist., Doi Khun Tan, 523 m, 8. xii.2007, H. Kurahashi (IDD); $10^{7}$, Chiang Mai Prov., Doi SuthepPui National Park, Sirindhorn Observatory, 817 m, 27.vii.2007, H. Kurahashi (IDD); $30^{\top}$, Chiang Mai Prov., Muang, Doi Suthep-Pui Mount, Tham Phra Leusri, 950 m, 11.iii.2011, H. Kurahashi (IDD); $40^{\top} 1$ 우, Chiang Mai Prov., Doi Suthep-Pui National Park, Sirindhorn Observatory, 817 m, 11.iii.2011, H. Kurahashi (IDD); 14\%? Chiang Mai Prov., Doi Saket, Doi Nang Kaew, 1,016 m, 10.iii.2011,

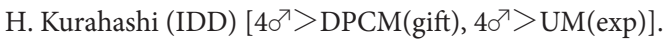

\section{Sarcorohdendorfia inextricata (Walker, 1859)}

THAILAND: $10^{\top}$, Chiangmai Prov., Chiangmai, 9.ix.1989, H. Kurahashi (NIID); $10^{\top}$, Surat Thani Prov., Ko Samui, N. Hinlad, A2, 20 21.iv.1988, H. Kurahashi (NIID); $10^{\top}$, Chiang Mai Prov., Mae kuang dam, 26.x.2000, S. Lertthamnongtham (DPCM); $10^{7}$, Chiang Mai Prov., Hang Dong Dist., Samoeng-Hang Dong rd., 390 m, 13. iii.2007, H. Kurahashi (IDD); $10^{7}$, Chiang Mai Prov., Hang Dong Dist., Samoeng-Hang Dong rd., 390 m, 9.xii.2007, H. Kurahashi (IDD); $60^{\top} 2$ 우, Chiang Mai Prov., Muang, Doi Suthep-Pui, $918 \mathrm{~m}$,

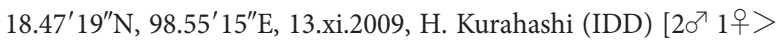
$\mathrm{UM}(\exp ), 4 \sigma^{\nearrow} 1$ 우> NSMT(gift)]; $1 \sigma^{\nearrow}$, Chiang Mai Prov., Doi Saked, Ban Pangdoeng, 508 m, 25.vii.2007, H. Kurahashi (IDD); $10^{\top}$, Chiang Mai Prov., Doi Suthep-Pui National Park, Sirindhorn Observa-

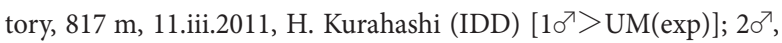
Chiang Mai Prov., Hang Dong Dist., Huay Mae Khanin, 552 m, 13.iii.2007, H. Kurahashi (IDD) [20 $>\mathrm{DPCM}$ (gift)]; $10^{\top}$, Chiang Mai Prov., Doi Suthep-Pui National Park, Sirindhorn Observatory, 817 m, 11.iii.2011, H. Kurahashi (IDD) [10 $>\mathrm{UM}(\exp )]$.

Sarcorohdendorfia montana Shinonaga \& Tumrasvin, 1979

THAILAND: 10 $\sigma^{\top}$, Prachuabkirikhan, 16.vii.1963,? (NIID); 30',

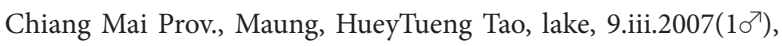
3.xii.2007 (207), H. Kurahashi (IDD).

\section{Sarcorohdendorfia multivillosa Shinonaga \& Tumras-} vin, 1979

THAILAND: $10^{7}$, Chiang Mai Prov., Doi Suthep-Pui National Park, Sun Khu, Doi Pui Summit, 1,587 m, 4.iii.2007, T. Chaiwong (IDD) $\left[10^{\top>N S M T(g i f t)] ; ~} 1 \sigma^{\nearrow}\right.$, Chiang Mai Prov., Chom Thong, Doi Inthanon, Ban Mae Klang, 924 m, 12.iii.2007, H. Kurahashi (IDD); $2 \sigma^{\top}$, Chiang Mai Prov., Doi Saket, Doi Nang Kaew, 1,142 m, $19.03^{\prime} 53^{\prime \prime} \mathrm{N}, 99.22^{\prime} 34^{\prime \prime} \mathrm{E}, 14 . x i .2009$, T. Klongklaew and H. Kurahashi (IDD) [20 $>$ NSMT(gift)]; $2 \sigma^{\top}$, Chiang Mai Prov., Doi Saket, Doi Nang Kaew, 972 m, 7-8.iii.2007, H. Kurahashi (IDD) $\left[10^{\top}>\mathrm{NSMT}\right.$ (gift); $\left.10^{\top}>\mathrm{UM}(\exp )\right] ; 30^{\top}$, Chiang Mai Prov., Doi Suthep-Pui National Park, Sirindhorn Observatory, 817 m, 7. xii.2007, H. Kurahashi (IDD); $30^{7} 1$ 우, Chiang Mai Prov., Doi Saket, Doi Nang Kaew, 1,016 m, 10.iii.2011, H. Kurahashi (IDD) $\left[10^{\top}>\mathrm{UM}(\exp )\right] ; 10^{\top}$, Chiang Mai Prov., Doi Saket District, Doi Nang Kaew, 19.03'53"N, 99.22'34"E, 1,142 m, 13.xi.2011, H. Kurahashi (IDD); $10^{\top}$, Chiang Mai Prov., Doi Suthep-Pui Mt., Siridhrn Observatory, 817 m, 15.xi.2011, H. Kurahashi (IDD); $3 \sigma^{7}$ 1우, Chiang Mai Prov., Doi Saket, Doi Nang Kaew, 1,016 m,

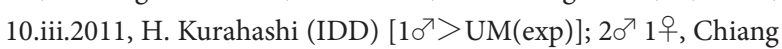
Mai Prov., Doi Suthep-Pui Mt., Tham Phra Leusri, 15.xi.2011, H. Kurahashi (IDD).
Sarcorohdendorfia seniorwhitei (Ho, 1938)

THAILAND: $10^{\top}$, Khao Yai, 12.ii.1964,? (NIID); $10^{7} 1$ 우, Chiang Mai Prov., Doi Inthanon, 1,300 m, A7, 10-12.v.1988, H. Kurahashi (NIID); $30^{\top} 1$ 우, Chiang Mai Prov., Doi Suthep-Pui National Park, Sirindhorn Observatory, $817 \mathrm{~m}, 4-5.1 i i .2007\left(10^{7}\right), 27$.

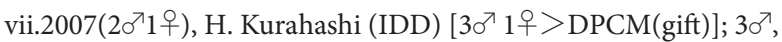
Chiang Mai Prov., Doi Suthep-Pui National Park, Sirindhorn Observatory, $817 \mathrm{~m}$, 11.iii.2011, H. Kurahashi (IDD) $\left[20^{\top \top}>\right.$ UM(exp)]; 10 $0^{\Upsilon}$, Chiang Mai Prov., Muang, Doi Suthep-Pui Mount, Tham Phra Leusri, 950 m, 11.iii.2011, H. Kurahashi (IDD); 1우, Lampang Prov., Hang Chat Dist., Doi Khun Tan rest area, 523 m, 28.vii.2007, H. Kurahashi (IDD); $10^{\gamma}$, Chiang Mai Prov., Doi Saked, Doi Nang Kaew, 972 m, 7-8.iii.2007, H. Kurahashi (IDD); $10^{7}$, Chiang Mai Prov., Doi Saket, Doi Nang Kaew, 1,016 m, 10.iii.2011, H. Kurahashi (IDD); 1우, Chiang Mai Prov., Doi Suthep-Pui Mt., Tham Phra Leusri, 15.xi.2011, H. Kurahashi (IDD); $30^{7} 1$ 우, Chiang Mai Prov., Doi Suthep-Pui Mt., Siridhorn Observatory, 817 m, 15.xi.2011, H. Kurahashi (IDD); 10', Chiang Mai Prov., Doi Saket, Doi Nang Kaew, 1,016 m, 10.iii.2011, H. Kurahashi (IDD); $30^{\top}$, Chiang Mai Prov., Doi Suthep-Pui National Park, Sirindhorn Observatory, 817 m, 11.iii.2011, H. Kurahashi (IDD) $\left[2 \sigma^{\top}>\mathrm{UM}(\exp )\right] ; 1 \sigma^{\top}$, Chiang Mai Prov., Muang, Doi Suthep-Pui Mount, Tham Phra Leusri, 950 m, 11.iii.2011, H. Kurahashi (IDD); $10^{\top} 1$ 우, Chiang Mai Prov., Doi Saket District, Doi Nang Kaew, $19.03^{\prime} 53^{\prime \prime} \mathrm{N}, 99.22^{\prime} 34^{\prime \prime} \mathrm{E}, 1,142$ m, 13.xi.2011, H. Kurahashi (IDD).

Sarcosolomonia aureomarginata Shinonaga \& Tumrasvin, 1979

Sarcosolomonia aureomarginata Shinonaga \& Tumrasvin, 1979: 140.

Sarcosolomonia circa (Pape \& Bänziger, 2003)

Sarcophaga (Sarcosolomonia) circa Pape \& Bänziger, 2003: 50 .

Sarcosolomonia crinita (Parker, 1917)

THAILAND: $10^{7}$, Chiang Mai Prov., Doi Saket, Doi Nang Kaew, 1,142 m, 14.xi.2009, H. Kurahashi (IDD) [1 $\left.10^{\top}>\mathrm{UM}(\exp )\right]$.

\section{Sarcosolomonia rohdendorfi Nandi, 1976}

THAILAND: $10^{\top}$, Surat Thani Prov., Ko Samui, N. Hinlad, A2, 20-21.iv.1988, H. Kurahashi (NIID); $30^{\nearrow}$, Surat Thani Prov., Khlong Sa, A4, 22.iv.1988, H. Kurahashi (NIID); $20^{\nearrow}$, Chon Buri Prov., Pattaya, A5, nr beach, 5.v.1988, H. Kurahashi (NIID); $2 \sigma^{7}$, Chiang Mai Prov., Doi Inthanon, 1,300 m, A7, 10-12.v.1988, H. Kurahashi (NIID); 5 우, Chiang Mai Prov., Chieng Mai City, riverside, 11.ii.2003, H. Kurahashi (IDD); 10 $\sigma^{\top}$, Chiang Mai Prov., Chi-

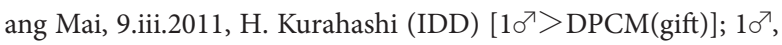
Chiang Mai Prov., Doi Saket, Doi Nang Kaew, 972 m, 7-8.iii.2007, H. Kurahashi (IDD) $\left[10^{\top}>\mathrm{UM}(\exp )\right]$; 1 우, Chiang Mai Prov., Chom Thong, Doi Inthanon, 1,494 m, 18.30'52"N, 98.31'33", 11.xi.2009, H. Kurahashi (IDD) [1우> UM(exp)]; $1 \sigma^{\prime}$, Chiang Mai Prov., Doi Saket, Doi Nang Kaew, 1,016 m, 10.iii.2011, H. Kurahashi (IDD); $10^{7}$, Chiang Mai Prov., Doi Suthep-Pui National Park, Sirindhorn Observatory, 817 m, 11.iii.2011, H. Kurahashi (IDD); $40^{7} 4$ 우, Chiang Mai Prov., Saraphi Dist., T. Thawangtan, $18.43^{\prime} 08^{\prime \prime} \mathrm{N}, 98.59^{\prime} 13^{\prime \prime} \mathrm{E}, 315$ m, 9.iii.2011, H. Kurahashi (IDD) 
$\left[30^{\top}>\mathrm{UM}(\exp )\right] ;$ 1우, Chiang Mai Prov., Doi Saket, Ban Pang Deang, Nam Mae Kuang Bridge, 551 m, 7-8.iii.2007, H. Kurahashi (IDD) [1우>NSMT(gift)]; 307, Chiang Mai Prov., Doi Suthep-Pui National Park, Sirindhorn Observatory, 817 m, 27. vii.2007, H. Kurahashi (IDD); $10^{7} 2$ 우, Chiang Mai Prov., Maung, HueyTueng Tao, lake, 9.iii.2007, H. Kurahashi (IDD) [2우> BPBM(gift)]; $20^{7} 3$ 우, Chiang Mai Prov., Doi Saked-Ban Pang Deang, Nam Mae Kuang Bridge, 551 m, 7-8.iii.2007, H. Kurahashi (IDD) [3우>BPBM(gift)]; $20^{\top} 4$ 우, Chiang Mai Prov., Hang Dong Dist., Samoeng-Hang Dong rd., 390 m, 13.iii.2007, H. Kurahashi (IDD) [4우>DPCM(gift)]; $20^{7}$, Chiang Mai Prov., Hang Dong Dist., T. Nongkaeo, $18.40^{\prime} 52^{\prime \prime} \mathrm{N} 98.55^{\prime} 57^{\prime \prime} \mathrm{E}, 360 \mathrm{~m}$, 9.iii.2011, H. Kurahashi (IDD); $50^{\top} 1$ 우, Chiang Mai Prov., Doi Saked, Ban Pangdoeng, 508 m, 25.vii.2007, H. Kurahashi (IDD); $7 \sigma^{7} 1$ 우, Lampang Prov., Hang Chat Dist., Doi Khun Tan, rest area, 523 m, 28.vii.2007, H. Kurahashi (IDD) [7 $7 \sigma^{\top}>\mathrm{BPBM}$ (gift)]; $2 \sigma^{\top}$, Lampang Prov., Hang Chat Dist., Doi Khun Tan, 523 m, 8. xii.2007, H. Kurahashi (IDD); $20^{\top}$, Lampang Prov., Hang Dong Dist., Samoeng-Hang Dong rd., 523 m, 8.xii.2007, H. Kurahashi (IDD); $40^{7} 4$ 우, Chiang Mai Prov., Saraphi Dist., T. Thawangtan, $18.43^{\prime} 08^{\prime \prime} \mathrm{N}, 98.59^{\prime} 13^{\prime \prime} \mathrm{E}, 315 \mathrm{~m}$, 9.iii.2011, H. Kurahashi (IDD)

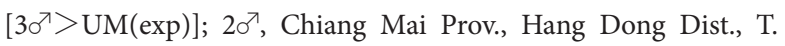
Nongkaeo, $18.40^{\prime} 52^{\prime \prime} \mathrm{N}, 98.55^{\prime} 57^{\prime \prime} \mathrm{E}, 360 \mathrm{~m}$, 9.iii.2011, H. Kurahashi (IDD); $1 \sigma^{\nearrow}$, Chiang Mai Prov., Doi Saket, Doi Nang Kaew, 1,016 m, 10.iii.2011, H. Kurahashi (IDD); 10 , Chiang Mai Prov., Chiang Mai, 9.iii.2011, H. Kurahashi (IDD) [1 $10^{\Upsilon}>$ DPCM(gift)]; $10^{\top}$, Chiang Mai Prov., Doi Suthep-Pui National Park, Sirindhorn Observatory, 817 m, 11.iii.2011, H. Kurahashi (IDD); $10^{7}$, Ubon Ratchathani Prov., Muang, Paddy field, $15^{\circ} 18^{\prime} 2.562^{\circ} \mathrm{N}, 104^{\circ} 52^{\prime}$ 50.035 ${ }^{\circ}$ E, $120 \mathrm{~m}, 13 . i i .2011$, T. Chaiwong (CMPH).

Sarcosolomonia shinonagai Kano \& Sooksri, 1977 THAILAND: 10 $0^{\top}$, Kancahnabri, 4.ix.1964,? (IDD).

Sarcosolomonia trifulcata Shinonaga \& Tumrasvin, 1979

Sarcosolomonia trifulcata Shinonaga \& Tumrasvin, 1979: 140.

The postsutural $d c 5$ group

\section{Alisarcophaga gressitti (Hall \& Bohart, 1948)}

THAILAND: $4 \sigma^{\top}$, Surat Thani Prov., Ko Samui, Tong Yang, A3, 22.iv.1988, H. Kurahashi (NIID).

\section{Asceloctella calicifera (Böttcher, 1912)}

THAILAND: $10^{\gamma} 1$ 우, Chiang Mai Prov., Hang Dong Dist., T. Nongkaeo, $18.40^{\prime} 52^{\prime \prime} \mathrm{N}, 98.55^{\prime} 57^{\prime \prime} \mathrm{E}, 360$ m, 9.iii.2011, H. Kurahashi (IDD) $\left[10^{\top}>\mathrm{UM}(\exp )\right]$; 1 우, Chiang Mai Prov., Muang Dist., T. Chang Puek, Nong Ho, 4.ix.2009, K. Moophayak (DPCM); 10', Chiang Mai Prov., Saraphi Dist., T. Thawangtan, 18.43'08"N 98.59'13"E, 315 m, 9.iii.2011, K. Moophayak (DPCM); 10', Chiang Mai Prov., Saraphi Dist., T. Thawangtan, $18.43^{\prime} 08^{\prime \prime} \mathrm{N} 98.59^{\prime}$ $13^{\prime \prime} \mathrm{E}, 315 \mathrm{~m}$, 9.iii.2011, K. Moophayak (DPCM); $10^{\top} 1$ 우, Chiang Mai Prov., Hang Dong Dist., T. Nongkaeo, $18.40^{\prime} 52^{\prime \prime} \mathrm{N}, 98.55^{\prime} 57^{\prime \prime}$ E, 360 m, 9.iii.2011, H. Kurahashi (IDD) [10 $>\mathrm{UM}(\exp )]$.
Bercaea africa (Wiedemann, 1824)

THAILAND: $30^{7}$, Chiang Mai Prov., Doi Inthanon, 1,300 m, 1012.v.1988, H. Kurahashi (NIID); $10^{\top}$, Chiang Mai Prov., Chom Thong, Doi Inthanon, 2,147 m, 12.iii.2007, H. Kurahashi (IDD); $10^{7}$, Chiang Mai Prov., Chom Thong, Doi Inthanon, 1,494 m, 11.xi.2009, H. Kurahashi (IDD) [10 $>\mathrm{UM}(\exp )]$.

Boettcherisca highlandica Kurahashi \& Tan, 2009 THAILAND: $10^{\top}$, Chiang Mai Prov., Muang, Doi Suthep-Pui, 18.47' $19^{\prime \prime} \mathrm{N}, 98.55^{\prime} 15^{\prime \prime}$ E, 13.xi.2009, H. Kurahashi (IDD); 10', Chiang Mai Prov., Doi Suthep-Pui National Park, Sirindhorn Observatory, 817 m, 27.vii.2007, H. Kurahashi (IDD).

\section{Boettcherisca javanica Lopes, 1961}

THAILAND: 10', Bangkok, Bangna Nai, 28.viii.1975, H. Kurahashi (NSMT); $10^{\top}$, Chon Buri, Salt Pond, 6 km N, 23.xii.1975, W. Tumrasvin (NSMT); 10 , Chon Buri, mangrove, 23.xii.1975, S. Shinonaga (NSMT); 50', Bangkok, Mahidol Univ., 4.ix.1975, H. Kurahashi (NSMT); 10', Chon Buri, mangrove, 23.xii.1975, S. Shinonaga (NSMT). KO SAMUI I.: $2 \sigma^{\top}$, Surat Thani Prov., nr Wat Nok, Khlong Sa, A4, 22.iv.1988, H. Kurahashi (NIID); 7o', Surat Thani Prov., Ko Samui, N. Hinlad, A2, 20-21.iv.1988, H. Kurahashi (NIID).

Boettcherisca krathonmai (Pape \& Bänziger, 2000) THAILAND: $10^{\top} \mathrm{PT}$, Ranong Prov., Khlong Naka WL Sanctuary, Phya Nak Falls, 460 m, 31.i.1999, H. Bänziger (SMNH).

\section{Boettcherisca nathani Lopes, 1961}

THAILAND: $1 \sigma^{\gamma}$, Chiang Mai Prov., Mae kuang dam, 20.vii.2000, S. Lertthamnongtham (DPCM); $10^{\top}$, Chiangmai Prov., Doi Sthep, 1,000 m, 16.vii.1989, H. Kurahashi (NIID); 30 ' Chiang Mai Prov., Doi Sthep, 1,000 m, A6, 9-15.v.1988, H. Kurahashi (NIID); 20 Chiang Mai Prov., Mae Thang, hill side,10.ii.2003, H. Kurahashi (IDD) $\left[20^{\top}>\mathrm{DPCM}\right]$; $10^{\top} 1$ 우, Khao Yai, $30 \mathrm{~km} \mathrm{~S}$. of Pak Chong, det. as Sarcophaga nathani by E. Sugiyama, 1987, 25-26.xii.1975, S. Shinonaga (NSMT); $7 \sigma^{\top}$, Chiang Mai Prov., Doi Inthanon, 1,300 m, A7, 10-12.v.1988, H. Kurahashi (NIID); 50 1우, Chiang Mai Prov., Doi Inthanon, 1,300 m, A7, 10-12.v.1988, H. Kurahashi (NIID); $20^{7}$, Chiang Mai Prov., Doi Suthep-Pui National Park, Headquarters area, 1,075 m, 7.xii.2007, H. Kurahashi (IDD); $4 \sigma^{\top}$, Chiang Mai Prov., Maung, HueyTueng Tao, lake, 23.

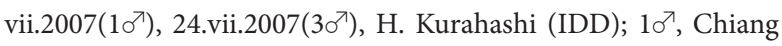
Mai Prov., Doi Saked, Doi Nang Kaew, 1,010 m, 25.vii.2007, H. Kurahashi (IDD); $10^{7}$, Lampang Prov., Hang Chat Dist., Doi Khun Tan rest area, $523 \mathrm{~m}$ 28.vii.2007, H. Kurahashi (IDD); $50^{7}$ 1우, Chiang Rai Prov., Pa Tuena Dist., Mae Chan, Huai Mahin Fon fall, 500 m, 5.xii.2007, H. Kurahashi (IDD); $2 \sigma^{7}$, Chiang Mai Prov., Doi Suthep-Pui National Park, Sirindhorn Observatory, 817 m, 4-5.iii.2007, H. Kurahashi (IDD); $30^{7}$ 2우, Chiang Mai Prov., Doi Suthep-Pui National Park, Sirindhorn Observatory, 817 m, 7.xii.2007, H. Kurahashi (IDD); $60^{7} 1$ 우, Chiang Mai Prov., Hang Dong Dist., Huay Mae Khanin, 552 m, 13.iii.2007, H. Kurahashi (IDD) $\left[6 \sigma^{\gamma} 1\right.$ 우>DPCM(gift)]; $14 \sigma^{\top}$, Lampang Prov., Hang Chat Dist., Doi Khun Tan, 523 m, 8.xii.2007, H. Kurahashi (IDD) $\left[7 \sigma^{\curlyvee}>\mathrm{BPBM}\right.$ (gift), $70^{\top}>\mathrm{BMNH}$ (gift)]; $7 \sigma^{\curlyvee} 2$ 우, Chiang Mai Prov., Doi Saked, Doi Nang Kaew, 1,010 m, 26.vii.2007, H. Kurahashi 
(IDD) $\left[5 \sigma^{7}\right.$ 2우>CMNH(gift)]; 5 $7 \sigma^{7}$, Chiang Mai Prov., Hang Dong Dist., Samoeng-Hang Dong rd., 390 m, 9.xii.2007, H. Kurahashi (IDD); $10^{\top}$, Chiang Mai Prov., Doi Saked, Doi Nang Kaew, 1,016 m, 6.xii.2007, H. Kurahashi (IDD); 2우, Chiang Mai Prov., Chieng Mai, hill side, 6.ii.2003, H. Kurahashi (IDD); 90 1우, Chiang Mai Prov., Hang Dong Dist., Samoeng-Hang Dong rd., 390 m, 13.iii.2007, H. Kurahashi (IDD) [ZMUC]; 40 7 우, Chiang Mai Prov., Muang, Doi Suthep-Pui, 918 m, 18.47'19"N, 98.55'15" E, 13.xi.2009, H. Kurahashi (IDD) [20 20우 $>$ NSMT(gift), $2 \sigma^{\top} 3$ 우 $>\mathrm{UM}(\exp )] ; 1 \sigma^{\top} 3$ 우, Chiang Mai Prov., Doi Saked-Ban Pang Deang, Nam Mae Kuang Bridge, 551 m, 7-8.iii.2007, H. Kurahashi (IDD); $10^{7} 1$ 우, Khao Yai, $30 \mathrm{~km} \mathrm{~S}$. of Pak Chong, det. as Sarcophaga nathani by E. Sugiyama, 1987, 25-26.xii.1975, S. Shinonaga (NSMT); $10^{\gamma}$, Chiang Rai Prov., Mae Suav District, $461 \mathrm{~m}$, 6.xii.2007, H. Kurahashi (IDD); 5 $\sigma^{\top}$, Chiang Mai Prov., Doi Saket, Doi Nang Kaew, 1,016 m, 10.iii.2011, H. Kurahashi (IDD); 9 $0^{\top}$, Chiang Mai Prov., Muang, Doi Suthep-Pui Mount, Tham Phra Leusri, 950 m, 11.iii.2011, H. Kurahashi (IDD); 90', Chiang Mai Prov., Doi Suthep-Pui National Park, Sirindhorn Observatory, 817 m, 11.iii.2011, H. Kurahashi (IDD) [30 $>\mathrm{UM}(\exp )]$; $10^{\top}$, Chiang Mai Prov., Chom Thong, Doi Inthanon, Ban Mae Klang, 924 m, 12.iii.2007, H. Kurahashi (IDD) [10 $>$ NSMT(gift)]; $20^{x}$ 1우, Chiang Mai Prov., Doi Saket, Doi Nang Kaew, 1,142 m, 19.03' $53^{\prime \prime} \mathrm{N}, 99.22^{\prime} 34^{\prime \prime} \mathrm{E}, 14 . x i .2009$, H. Kurahashi (IDD) [20 $10^{\top}$ 우>

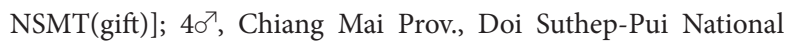
Park, Doi Pui Summit-Sun Khu, 1,587 m, 4-5.iii.2007, H. Kurahashi (IDD); $10^{\gamma}$, Chiang Rai Prov., Pa Tuena Dist., Mae Chan, Laan Tong resort, 449 m, 4.xii.2007, H. Kurahashi (IDD); 90', Chiang Mai Prov., Doi Suthep-Pui National Park, Sirindhorn Observatory, $817 \mathrm{~m}, 11$. iii.2011, H. Kurahashi (IDD) $\left[30^{\top}>\right.$ UM(exp)]; 90 , Chiang Mai Prov., Muang, Doi Suthep-Pui Mount, Tham Phra Leusri, 950 m, 11.iii.2011, H. Kurahashi (IDD); $10^{7}$ 1우, Chiang Mai Prov., Mae Taeng Dist., Mae Taeng Elephant Camp, 345 m, 14.xi.2011, H. Kurahashi (IDD); 50', Chiang Mai Prov., Doi Saket, Doi Nang Kaew, 1,016 m, 10.iii.2011, H. Kurahashi (IDD); $80^{\top}$ 6우, Chiang Mai Prov., Doi Saket, Doi Nang Kaew, 13.xi.2011, H. Kurahashi (IDD) $\left[10^{\top}>\operatorname{DPCM}(\right.$ gift) $]$; $60^{7}$ 2우, Chiang Mai Prov., Doi Suthep-Pui Mt., Tham Phra Leusri, 15.xi.2011, H. Kurahashi (IDD). SAMET IS.: 107, Rayon, 26. viii.1986, Y. Wada (NIID).

Boettcherisca peregrina (Robineau-Desvoidy, 1830) THAILAND: $2 \sigma^{\top}$, Chiang Mai Prov., Hang Dong, 13.ii.2003, K. Sukontason (DPCM); 12ð', Bangkok, Mahidol Univ., 2.ix.1975

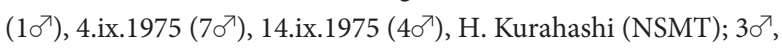
Ayutthaya, woods \& grass ld., 1.ix.1975, H. Kurahashi (NSMT); $5 \sigma^{\gamma}$, Chiang Mai Prov., Chiang Mai, river side, 11.ii.2003, H. Kurahashi (IDD) $\left[50^{\top}>\right.$ DPCM]; $20^{\top}$, Chon Buri, Salt Pond $6 \mathrm{~km}$ N, 2.ix.1975, H. Kurahashi (NSMT); 1우, Chiang Mai Prov., Muang Tha, Sala, 13.ii.2003, H. Kurahashi (IDD) [1우>DPCM]; $2 \sigma^{7}$, Bangkok, Bangna Nai, 28.viii.1975, H. Kurahashi (NSMT); $10 \sigma^{7}$, Bangkok, Sam Sane, 29.viii.1975, R. Kano, H. Kurahashi and W. Tumrasvin (NSMT); 1우, Chiang Mai Prov., Hang Dong, Nong Thang, 14.ii.2003, H. Kurahashi (IDD) [1우>DPCM]; 30 , nr. Ban Sap Bon, 30 km E Sara Buri, 4.x.1975, R. Kano (NSMT); 2疋, Bangkok, Bangna Nai, 28.viii.1975, H. Kurahashi (NSMT); $2 \sigma^{\top}$, Chon Buri, Salt Pond 6 km N, 2.ix.1975, H. Kurahashi (NSMT);
$3 \sigma^{7}$, Ayutthaya, woods \& grass ld., 1.ix.1975, H. Kurahashi

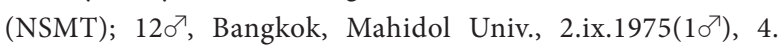

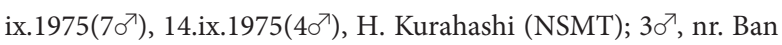
Sap Bon, 30 km E Sara Buri, 4.x.1975, R. Kano (NSMT); 10 7 , Bangkok, Sam Sane, 29.viii.1975, R. Kano, H. Kurahashi and W. Tumrasvin (NSMT); 1우, Chiang Rai Prov., Pa Tuena Dist., Mae Chan, Huai Mahin Fon fall, 500 m, 5.xii.2007, H. Kurahashi (IDD); 1 우, Chiang Mai Prov., Hang Dong Dist., Samoeng-Hang Dong rd., 390 m , 13.iii.2007, H. Kurahashi (IDD); $110^{7} 1$ 우, Chiang Mai Prov., Ping river, San Sai, 9.iii.2007, H. Kurahashi (IDD) [7 $\left.0^{\nearrow}>\operatorname{DPCM}(\mathrm{gift})\right] ; 80^{\nearrow}$, Chiang Mai Prov., Hang Dong Dist., T. Nongkaeo, $18.40^{\prime} 52^{\prime \prime} \mathrm{N}, 98.55^{\prime} 57^{\prime \prime} \mathrm{E}, 360 \mathrm{~m}$, 9.iii.2011, H. Kurahashi (IDD) $\left[3 \sigma^{\top}>\operatorname{DPCM}\left(\right.\right.$ gift)]; $90^{\top}$, Chiang Mai Prov., Saraphi Dist., T. Thawangtan, $18.43^{\prime} 08^{\prime \prime} \mathrm{N}, 98.59^{\prime} 13^{\prime \prime} \mathrm{E}, 315 \mathrm{~m}$, 9.iii.2011, H. Kurahashi (IDD), [30 $>\mathrm{UM}(\exp )]$; $80^{\top}$, Chiang Mai Prov., Hang Dong Dist., T. Nongkaeo, 18.40'52"N, 98.55'57"E, 360 m,

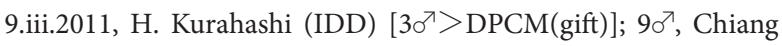
Mai Prov., Saraphi Dist., T. Thawangtan, $18.43^{\prime} 08^{\prime \prime} \mathrm{N}, 98.59^{\prime} 13^{\prime \prime} \mathrm{E}$, 315 m, 9.iii.2011, H. Kurahashi (IDD) $\left[30^{\top}>\mathrm{UM}(\exp )\right]$; $80^{\top}$, Ubon Ratchathani Prov., Muang, Paddy field, $15^{\circ} 18^{\prime} 2.562^{\circ} \mathrm{N}, 104^{\circ}$ 52'50.035 ${ }^{\circ}$ E, 120 m, 23.i.2011, 13.iii.2011, 12.vi.2011, 7.vii.2011, T. Chaiwong (CMPH); $20^{\top}$, Ubon Ratchathani Prov., Warinchamrap, Paddy field, $15^{\circ} 8^{\prime} 21.388^{\circ} \mathrm{N}, 104^{\circ} 53^{\prime} 52.218^{\circ} \mathrm{E}, 137 \mathrm{~m}, 7$. vii.2011, T. Chaiwong (CMPH); $10^{7}$, Ubon Ratchathani Prov., Warinchamrap, Restaurant, $15^{\circ} 7^{\prime} 43.603^{\circ} \mathrm{N}, 104^{\circ} 54^{\prime} 0.579^{\circ} \mathrm{E}, 145 \mathrm{~m}$, 2.ii.2011, T. Chaiwong (CMPH); $10^{7}$, Ubon Ratchathani Prov., Warinchamrap, School cafeteria, $15^{\circ} 11^{\prime} 33.212^{\circ} \mathrm{N}, 104^{\circ} 50^{\prime} 6.756^{\circ} \mathrm{E}$, 126 m, 12.iii.2011, T. Chaiwong (CMPH).

\section{Harpagophalla kempi (Senior-White, 1924)}

THAILAND: $50^{\nearrow}$, Chiang Mai Prov., Doi Inthanon, 1,300 m, A7, 10-12.v.1988, H. Kurahashi (NIID); $10^{7}$, Surat Thani Prov., Ko Samui, N. Hinlad, A2, 20-21.iv.1988, H. Kurahashi (NIID); 10 Surat Thani Prov., nr Wat Nok, Khlong Sa, A4, 22.iv.1988, H. Kurahashi (NIID); $60^{\top}$, Tak Prov., $80 \mathrm{~km}$ NW of Mae Sot, Mae Rayang, 9.vi.1988, H. Kurahashi (NIID); $1 \sigma^{\top}$, Chiang Mai Prov., Doi Sthep, 1,000 m, A6, 9-15.v.1988, H. Kurahashi (NIID); 10?' Chiang Mai Prov., Doi Suthep-Pui National Park, Headquarters area, 1,075 m, 4-5.iii.2007, H. Kurahashi (IDD) $\left[10^{\top}>\right.$ DPCM(gift)]; $10^{\top}$, Chiang Mai Prov., Hang Dong Dist., SamoengHang Dong rd., 390 m, 9.xii.2007, H. Kurahashi (IDD) $\left[10^{7}>\right.$ DPCM(gift)]; $1 \sigma^{\top}$, Chiang Mai Prov., Saraphi Dist., T. Thawang$\tan , 18.43^{\prime} 08^{\prime \prime} \mathrm{N}, 98.59^{\prime} 13^{\prime \prime} \mathrm{E}, 315 \mathrm{~m}$, 9.iii.2011, H. Kurahashi (IDD) $\left[10^{\top}>\mathrm{UM}(\exp )\right]$; $10^{7}$, Chiang Mai Prov., Doi Saket, Doi Nang Kaew, 972 m, 7-8.iii.2007, H. Kurahashi (IDD) $\left[10^{\top>}\right.$ UM(exp)]; $10^{7}$, Chiang Mai Prov., Doi Saked-Ban Pang Deang, Nam Mae Kuang Bridge, 551 m, 7-8.iii.2007, H. Kurahashi (IDD) $\left[10^{\top}>\operatorname{DPCM}(\mathrm{gift})\right]$; $10^{\nearrow}$, Chiang Mai Prov., Ping river, San Sai, 9.iii.2007, H. Kurahashi (IDD) [10 ]; $10^{\curlyvee}$, Chiang Mai Prov., Hang Dong Dist., Samoeng-Hang Dong rd., 390 m, 13.iii.2007, H. Kurahashi (IDD) $\left[10^{\top}>\right.$ DPCM]; $10^{\top}$, Chiang Rai Prov., Pa Tuena Dist., Mae Chan, Laan Tong resort, 449 m, 4.xii.2007, H. Kura-

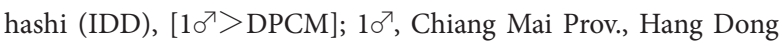
Dist., T. Nongkaeo, 18.40'52"N, 98.55'57"E, 360 m, 9.iii.2011, H. Kurahashi (IDD); $10^{7}$, Chiang Mai Prov., Saraphi Dist., T. Thawangtan, $18.43^{\prime} 08^{\prime \prime} \mathrm{N}, 98.59^{\prime} 13^{\prime \prime} \mathrm{E}, 315 \mathrm{~m}$, 9.iii.2011, H. Kurahashi (IDD) $\left[10^{\top}>\mathrm{UM}(\exp )\right]$; $50^{\Upsilon}$, Ubon Ratchathani Prov., Muang, 
Paddy field, $15^{\circ} 18^{\prime} 2.562^{\circ} \mathrm{N}, 104^{\circ} 52^{\prime} 50.035^{\circ} \mathrm{E}, 120 \mathrm{~m}, 13 . i i i .2011$, 12.vi.2011, 7.vii.2011, T. Chaiwong (CMPH); 10', Ubon Ratchathani Prov., Warinchamrap, School cafeteria, $15^{\circ} 11^{\prime} 33.212^{\circ} \mathrm{N}, 104^{\circ}$ $50^{\prime} 6.756^{\circ} \mathrm{E}, 126 \mathrm{~m}$, 2.iv.2011, T. Chaiwong (CMPH); $10^{\top}$, Ubon Ratchathani Prov., Warinchamrap, Garbage piles, $15^{\circ} 11^{\prime} 52.340^{\circ} \mathrm{N}$, $104^{\circ} 52^{\prime} 10.045^{\circ} \mathrm{E}, 133 \mathrm{~m}, 11 . v i .2011$, T. Chaiwong (CMPH); $2 \sigma^{\top}$, Ubon Ratchathani Prov., Warinchamrap, Paddy field, $15^{\circ} 8^{\prime} 21.388^{\circ}$ N, $104^{\circ} 53^{\prime} 52.218^{\circ} \mathrm{E}, 137 \mathrm{~m}, 2 . \mathrm{iv} .2011$, T. Chaiwong (CMPH); $10^{\top}$, Ubon Ratchathani Prov., Muang, Restaurant, $15^{\circ} 16^{\prime} 29.668^{\circ} \mathrm{N}, 104^{\circ}$ $49^{\prime} 27.471^{\circ} \mathrm{E}, 132 \mathrm{~m}, 11 . v i i i .2011$, T. Chaiwong (CMPH).

\section{Hosarcophaga serrata (Ho, 1938)}

Hosarcohaga serrata: Shinonaga \& Tumrasvin, 1979: 152.

\section{*Iranihindia martellatoides (Baranov, 1931)}

THAILAND: 10 $\sigma^{\top}$, Tak Prov., 80 km NW of Mae Sot, Mae Rayang, 9.iv.1988, H. Kurahashi (NIID).

\section{Liopygia ruficornis (Fabricius, 1794)}

THAILAND: 1 우, Chiang Mai Prov., Saraphi Dist., T. Thawang$\tan , 18.43^{\prime} 08^{\prime \prime} \mathrm{N}, 98.59^{\prime} 13^{\prime \prime} \mathrm{E}, 315$ m, 9.iii.2011, H. Kurahashi (IDD) [1우> UM(exp)]; 1우, Chiang Mai Prov., Chieng Mai City, 6.iii.2011, H. Kurahashi (IDD) [1우> UM(exp)]; 1우, Chiang Mai Prov., Chieng Mai City, 6.iii.2011, H. Kurahashi (IDD) [1우> $\mathrm{UM}(\exp )$ ]; 1 우, Chiang Mai Prov., Saraphi Dist., T. Thawangtan, $18.43^{\prime} 08^{\prime \prime} \mathrm{N}, 98.59^{\prime} 13^{\prime \prime} \mathrm{E}, 315$ m, 9.iii.2011, H. Kurahashi (IDD) [1우 $>\mathrm{UM}(\exp )]$; 1 우, Ubon Ratchathani Prov., Muang, Fresh-food market, $15^{\circ} 13^{\prime} 55.225^{\circ} \mathrm{N}, 104^{\circ} 52^{\prime} 22.748^{\circ} \mathrm{E}, 118 \mathrm{~m}, 13 . i i i .2011$, T. Chaiwong (CMPH); 4 우, Ubon Ratchathani Prov., Muang, Garbage piles, $15^{\circ} 15^{\prime} 35.620, \mathrm{~N} 104^{\circ} 50^{\prime} 55.601^{\circ} \mathrm{E}, 121 \mathrm{~m}, 12 . v i .2011$, 7.vii.2011, T. Chaiwong (CMPH); 1우, Ubon Ratchathani Prov., Muang, Paddy field, $15^{\circ} 18^{\prime} 2.562^{\circ} \mathrm{N}, 104^{\circ} 52^{\prime} 50.035^{\circ} \mathrm{E}, 120 \mathrm{~m}$, 13.iii.2011, T. Chaiwong (CMPH); 1우, Ubon Ratchathani Prov., Muang, Restaurant, $15^{\circ} 16^{\prime} 29.668^{\circ} \mathrm{N}, 104^{\circ} 49^{\prime} 27.471^{\circ} \mathrm{E}, 132 \mathrm{~m}$, 22.v.2011, T. Chaiwong (CMPH); $30^{7} 3$ 우, Ubon Ratchathani Prov., Muang, School cafeteria, $15^{\circ} 16^{\prime} 29.668^{\circ} \mathrm{N}, 104^{\circ} 49^{\prime} 27.471^{\circ} \mathrm{E}$, 127 m, 3.i.2011, 13.iii.2011, 22.v.2011, 12.vi.2011, 11.viii.2011, T. Chaiwong (CMPH); 1 우, Ubon Ratchathani Prov., Warinchamrap, Restaurant, $15^{\circ} 7^{\prime} 43.603^{\circ} \mathrm{N}, 104^{\circ} 54^{\prime} 0.579^{\circ} \mathrm{E}, 145 \mathrm{~m}, 12 . i i .2011$, T. Chaiwong (CMPH); 1 우, Ubon Ratchathani Prov., Warinchamrap, School cafeteria, $15^{\circ} 11^{\prime} 33.212^{\circ} \mathrm{N}, 104^{\circ} 50^{\prime} 6.756^{\circ} \mathrm{E}, 126 \mathrm{~m}$, 11.vi.2011, T. Chaiwong (CMPH); 5우, Ubon Ratchathani Prov., Warinchamrap, Garbage piles, $15^{\circ} 11^{\prime} 52.340^{\circ} \mathrm{N}, 104^{\circ} 52^{\prime} 10.045^{\circ} \mathrm{E}$, 133 m, 22.i.2011, 21.v.2011, 11.viii.2011, T. Chaiwong (CMPH); 1 우, Ubon Ratchathani Prov., Muang, Garbage piles, $15^{\circ} 15^{\prime} 35.620$, $\mathrm{N} 104^{\circ} 50^{\prime} 55.601^{\circ} \mathrm{E}, 121 \mathrm{~m}, 11$.viii.2011, T. Chaiwong (CMPH).

\section{Parasarcophaga albiceps (Meigen, 1826)}

THAILAND: $10^{7}$, Tak Prov., $65 \mathrm{~km}$ NE of Mae Sot, Ban Mai, 8.iv.1988, H. Kurahashi (NIID); $30^{\nearrow}$, Chiang Mai Prov., Doi Inthanon, 1,300 m, A7, 10-12.v.1988, H. Kurahashi (NIID); $2 \sigma^{7}$, Chiang Mai Prov., Doi Sthep, 1,000 m, A6, 9-15.v.1988, H. Kurahashi (NIID); $4 \sigma^{\top}$, Chiangmai Prov., Chiangmai, 9.ix.1989, H. Kurahashi (NIID); $10^{\gamma}$, Chiang Mai Prov., Doi Suthep-Pui National Park, Sirindhorn Observatory, 817 m, 27.vii.2007, H. Kurahashi (IDD) $\left[10^{\Upsilon}>\operatorname{DPCM}\right.$ (gift)]; $10^{\top}$, Lampang Prov., Hang Chat Dist.,
Doi Khun Tan rest area, 523 m, 28.vii.2007, H. Kurahashi (IDD) [1 $\left.1 \sigma^{\top}>\operatorname{DPCM}(\mathrm{gift})\right]$; $3 \sigma^{\top}$, Chiang Rai Prov., Pa Tuena Dist., Mae Chan, Huai Mahin Fon fall, 500 m, 5.xii.2007, H. Kurahashi (IDD) $\left[3 \sigma^{\top}>\operatorname{DPCM}(\right.$ gift) $]$; $5 \sigma^{\Upsilon}$, Chiang Mai Prov., Hang Dong Dist., Samoeng-Hang Dong rd., 390 m, 9.xii.2007, H. Kurahashi (IDD) $\left[50^{\top}>\mathrm{DPCM}(\right.$ gift) $]$; $10^{\top}$, Chiang Rai Prov., Maung, Khun Korn Fall, 630 m, 5.xii.2007, K. L. Sukontason (DPCM); 10 , Chiang Rai Prov., Pa Tuena Dist., Mae Chan, Huai Mahin Fon fall, 500 m, 5.xii.2007, H. Kurahashi (IDD) [10 $>$ DPCM(gift)]; 10 Chiang Mai Prov., Hang Dong Dist., Huay Mae Khanin, 552 m,

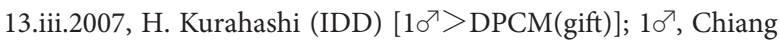
Mai Prov., Doi Saket, Doi Nang Kaew, 972 m, 7-8.iii.2007, H. Kurahashi (IDD) $\left[10^{\top}>\mathrm{NSMT}\left(\right.\right.$ gift)]; $50^{7}$, Chiang Mai Prov., Chom Thong, Doi Inthanon, 924 m, 12.iii.2007, H. Kurahashi (IDD) $\left[2 \sigma^{\top}>\mathrm{UM}(\exp ), 3 \sigma^{\top}>\mathrm{NSMT}\right.$ (gift)]; $10^{\top}$, Chiang Mai Prov., Doi Saket, Doi Nang Kaew, 1,142 m, 19.03'53"N, 99.22'34"E, 14.xi.2009, H. Kurahashi (IDD) [10 $>\mathrm{NSMT}$ (gift)]; $10^{\top}$, Chiang Mai Prov., Doi Saket, Ban Pang Deang, Nam Mae Kuang Bridge,

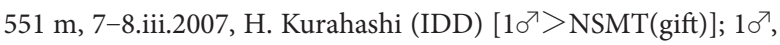
Chiang Mai Prov., Muang, Doi Suthep-Pui, $918 \mathrm{~m}, 18.47^{\prime} 19^{\prime \prime} \mathrm{N}$, 98.55'15"E, 13.xi.2009, H. Kurahashi (IDD) [1 $10^{\top}>\mathrm{NSMT}$ (gift)]; 1우, Chiang Mai Prov., Hang Dong Dist., T. Nongkaeo, 18.40'52" N, 98.55'57"E, 360 m, 9.iii.2011, H. Kurahashi (IDD); 1우, Chiang Mai Prov., Saraphi Dist., T. Thawangtan, $18.43^{\prime} 08^{\prime \prime} \mathrm{N}, 98.59^{\prime} 13^{\prime \prime} \mathrm{E}$, 315 m, 9.iii.2011, H. Kurahashi (IDD); 1우, Chiang Mai Prov., Hang Dong Dist., T. Nongkaeo, 18.40' $52^{\prime \prime} \mathrm{N}, 98.55^{\prime} 57^{\prime \prime} \mathrm{E}, 360 \mathrm{~m}$, 9.iii.2011, H. Kurahashi (IDD); 1우, Chiang Mai Prov., Saraphi Dist., T. Thawangtan, $18.43^{\prime} 08^{\prime \prime} \mathrm{N}, 98.59^{\prime} 13^{\prime \prime} \mathrm{E}, 315 \mathrm{~m}$, 9.iii.2011, H. Kurahashi (IDD). KO SAMUI I.: $90^{7}$, Surat Thani Prov., Ko Samui, N. Hinlad, A2, 20-22.iv.1988, H. Kurahashi (NIID).

Parasarcophaga amplicercus Shinonaga \& Tumrasvin, 1979

THAIlAND: $2 \sigma^{\top}$, Chiang Mai Prov., Doi Sthep, 1,000 m, A6, 9-15.v.1988, H. Kurahashi (NIID); 10', Chiang Rai Prov., Doi Saked-Ban Pang Deang, Nam Mae Kuang Bridge, 551 m, 7-8. iii.2007, H. Kurahashi (IDD).

Parasarcophaga bangkokensis (Shinonaga \& Tumrasvin, 1979)

Kanomyia bangkokensis Shinonaga \& Tumrasvin, 1979: 138.

\section{Parasarcophaga brevicornis (Ho, 1934)}

THAILAND: $10^{\top}$, Chiang Mai Prov., Mae aon, 16.xi.2000, S. Lertthamnongtham (DPCM); $10^{\top}$, Tak Prov., $80 \mathrm{~km} \mathrm{NW}$ of Mae Sot,

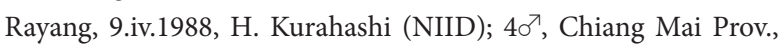
Saraphi Dist., T. Thawangtan, $18.43^{\prime} 08^{\prime \prime} \mathrm{N}, 98.59^{\prime} 13^{\prime \prime} \mathrm{E}, 315 \mathrm{~m}$, 9.iii.2011, H. Kurahashi (IDD) [1 $\left.10^{\top}>\mathrm{UM}(\exp )\right]$; $30^{\top}$, Chiang Rai Prov., Pa Tuena Dist., Mae Chan, Laan Tong resort, 449 m, 4. xii.2007, H. Kurahashi (IDD); $30^{\top}$, Chiang Mai Prov., Hang Dong Dist., Samoeng-Hang Dong rd., 390 m, 13.iii.2007, H. Kurahashi (IDD); $10^{\top}$, Chiang Rai Prov., Pa Tuena Dist., Mae Chan, Huai Mahin Fon fall, 500 m 5.xii.2007, H. Kurahashi (IDD); 90 4우, Chiang Mai Prov., Ping river, San Sai, 9.iii.2007, H. Kurahashi (IDD) $\left[7 \sigma^{\nearrow} 2\right.$ 우>DPCM(gift)]; $2 \sigma^{\nearrow}$, Chiang Mai Prov., Mae Taeng Dist., Mae Taeng Elephant Camp, 345 m, 14.xi.2011, H. Kura- 
hashi (IDD); $40^{\nearrow}$, Chiang Mai Prov., Saraphi Dist., T. Thawangtan, $18.43^{\prime} 08^{\prime \prime} \mathrm{N}, 98.59^{\prime} 13^{\prime \prime} \mathrm{E}, 315 \mathrm{~m}$, 9.iii.2011, H. Kurahashi (IDD) [1 $\left.10^{\top}>\mathrm{UM}(\exp )\right] ; 2 \sigma^{\top}$, Ubon Ratchathani Prov., Muang, Restaurant, $15^{\circ} 16^{\prime} 29.668^{\circ} \mathrm{N}, 104^{\circ} 49^{\prime} 27.471^{\circ} \mathrm{E}, 132 \mathrm{~m}, 12 . v i .2011,12$. iii.2011, T. Chaiwong (CMPH); $10^{\top}$, Ubon Ratchathani Prov., Warinchamrap, Restaurant, $15^{\circ} 7^{\prime} 43.603^{\circ} \mathrm{N}, 104^{\circ} 54^{\prime} 0.579^{\circ} \mathrm{E}, 145 \mathrm{~m}$, 11.viii.2011, T. Chaiwong (CMPH); $20^{\top}$, Ubon Ratchathani Prov., Warinchamrap, Fresh-food market, $15^{\circ} 10^{\prime} 40.369^{\circ} \mathrm{N}, 104^{\circ} 52^{\prime}$ 20.981 ${ }^{\circ}$, $121 \mathrm{~m}, 21 . v .2011$, T. Chaiwong (CMPH); $20^{\top}$, Ubon Ratchathani Prov., Warinchamrap, School cafeteria, $15^{\circ} 11^{\prime} 33.212$ ${ }^{\circ} \mathrm{N}, 104^{\circ} 50^{\prime} 6.756^{\circ} \mathrm{E}, 126 \mathrm{~m}, 12$. iii.2011, 11.vi.2011, T. Chaiwong $(\mathrm{CMPH}) ; 2 \sigma^{\top}$, Ubon Ratchathani Prov., Warinchamrap, Paddy field, $15^{\circ} 8^{\prime} 21.388^{\circ} \mathrm{N}, 104^{\circ} 53^{\prime} 52.218^{\circ} \mathrm{E}, 137 \mathrm{~m}, 12 . i i .2011,2$. iv.2011, T. Chaiwong (CMPH). KO SAMUI I.: $20^{\top}$, Surat Thani Prov., Ko Samui, N. Hinlad, A2, 20-21.iv.1988, H. Kurahashi (NIID).

\section{Parasarcophaga dux (Thomson, 1869)}

THAILAND: $3 \sigma^{\nearrow}$, Chiang Mai Prov., Doi Inthanon, 1,300 m, A7, 10-12.v.1988, H. Kurahashi (NIID); $2 \sigma^{\top}$, Chiang Mai Prov., Doi Sthep, 1,000 m, A6, 9-15.v.1988, H. Kurahashi (NIID); 80 , Chon Buri Prov., Pattaya, nr beach, A5, 5.v.1988, H. Kurahashi (NIID); $10^{\top}$, Tak Prov., 65 km NE of Mae Sot, Ban Mai, 8.iv.1988, H. Kurahashi (NIID); $10^{\text {T}}$, Tak Prov., $80 \mathrm{~km} \mathrm{NW}$ of Mae Rayang, 9. iv.1988, H. Kurahashi (NIID); 10 , Chiang Mai Prov., Mae aon, 16.xi.2000, S. Lertthamnongtham (DPCM); 10 , Surat Thani Prov., Khanom, beach, A1, 19.iv.1988, T. Phonchevin (NIID); $200^{\top} 2$ 우, Chiang Mai Prov., Hang Dong Dist., T. Nongkaeo, 18.40' $52^{\prime \prime} \mathrm{N}, 98.55^{\prime} 57^{\prime \prime} \mathrm{E}, 360 \mathrm{~m}$, 9.iii.2011, H. Kurahashi (IDD) [20 $70^{\top}>$ $\mathrm{UM}$ (exp), $30^{\top}>\mathrm{BPBM}$ (gift), $20^{\top}>\mathrm{PCCCHeo}$ (gift)]; $100^{\top} 1$ 우, Chiang Mai Prov., Ping river, San Sai, 9.iii.2007, H. Kurahashi (IDD) $\left[100^{7} 1\right.$ 우 $\left.>\mathrm{DPCM}(\mathrm{gift})\right]$; 1 우, Chiang Mai Prov., Doi Saked-Ban Pang Deang, 508 m, 25.vii.2007, H. Kurahashi (IDD); 1우, Chiang Mai Prov., Doi Saked, Doi Nang Kaew, 1,010 m, 25.vii.2007, H. Kurahashi (IDD); 1 우, Chiang Mai Prov., Doi Suthep-Pui National Park, Sirindhorn Observatory, 817 m, 27. vii.2007, H. Kurahashi (IDD); 1우, Lampang Prov., Hang Chat Dist., Doi Khun Tan rest area, 523 m, 28.vii.2007, H. Kurahashi (IDD); 1우, Chiang Rai Prov., Pa Tuena Dist., Mae Chan, Laan Tong resort, 449 m, 4.xii.2007, H. Kurahashi (IDD); 10 , Chiang Mai Prov., Doi Saket, Doi Nang Kaew, 972 m, 7-8.iii.2007, H. Kurahashi (IDD) [1 $\left.10^{7}>\mathrm{UM}(\exp )\right] ;$ 1우, Chiang Mai Prov., Saraphi Dist., T. Thawangtan, $18.43^{\prime} 08^{\prime \prime} \mathrm{N}, 98.59^{\prime} 13^{\prime \prime} \mathrm{E}, 315 \mathrm{~m}$, 9.iii.2011, H. Kurahashi (IDD) [1우>BPBM(gift)]; 9우, Chiang Mai Prov., Chieng Mai City, riverside, 11.ii.2003, H. Kurahashi (IDD); $20^{7}$ 1우, Chiang Mai Prov., Maung, HueyTueng Tao, lake, 9.iii.2007, H. Kurahashi (IDD) [2 $2 \sigma^{\top} 1$ 우>DPCM(gift)]; $2 \sigma^{\nearrow}$, Chiang Mai Prov., Hang Dong Dist., Samoeng-Hang Dong rd., 390 m, 13.iii.2007, H. Kurahashi (IDD); 3 우, Chiang Mai Prov., Doi Saked-Ban Pang Deang, Nam Mae Kuang Bridge, 551 m, 7-8.iii.2007, H. Kurahashi (IDD); 1우, Chiang Mai Prov., Hang Dong Dist., Huay Mae Khanin, 552 m, 13.iii.2007, H. Kurahashi (IDD); 200 구우, Chiang Mai Prov., Hang Dong Dist., T. Nongkaeo, 18.40'52"N, 98.55'57" E, $360 \mathrm{~m}$, 9.iii.2011, H. Kurahashi (IDD) [20 $20^{\top}>\mathrm{UM}(\exp ), 30^{\top}>$ $\mathrm{BPBM}$ (gift), $2 \sigma^{7}>\mathrm{PCCCHeo}$ (gift)]; 1우, Chiang Mai Prov., Saraphi Dist., T. Thawangtan, $18.43^{\prime} 08^{\prime \prime} \mathrm{N}, 98.59^{\prime} 13^{\prime \prime} \mathrm{E}, 315$ m, 9.iii.2011, H. Kurahashi (IDD) [1우>BPBM(gift)]; $2 \sigma^{\top}$, Ubon Ratchathani Prov.,
Muang, Paddy field, $15^{\circ} 18^{\prime} 2.562^{\circ} \mathrm{N}, 104^{\circ} 52^{\prime} 50.035^{\circ} \mathrm{E}, 120 \mathrm{~m}$, 22.v.2011, 12.vi.2011, T. Chaiwong (CMPH); $20^{\top}$, Ubon Ratchathani Prov., Warinchamrap, Paddy field, $15^{\circ} 8^{\prime} 21.388^{\circ} \mathrm{N}, 104^{\circ} 53^{\prime}$ $52.218^{\circ} \mathrm{E}, 137 \mathrm{~m}, 11 . v i .2011$, T. Chaiwong (CMPH); $10^{\top}$, Ubon Ratchathani Prov., Muang, Restaurant, $15^{\circ} 16^{\prime} 29.668^{\circ} \mathrm{N}, 104^{\circ} 49^{\prime}$ $27.471^{\circ} \mathrm{E}, 132 \mathrm{~m}, 22 . v \cdot 2011$, T. Chaiwong $(\mathrm{CMPH}) ; 20^{\top}$, Ubon Ratchathani Prov., Warinchamrap, Restaurant, $15^{\circ} 7^{\prime} 43.603^{\circ} \mathrm{N}$, $104^{\circ} 54^{\prime} 0.579^{\circ} \mathrm{E}, 145 \mathrm{~m}, 12$. ii.2011, 12.iii.2011, T. Chaiwong (CMPH) $10^{\top}$, Ubon Ratchathani Prov., Muang, School cafeteria, $15^{\circ} 16^{\prime} 29.668^{\circ} \mathrm{N}, 104^{\circ} 49^{\prime} 27.471^{\circ} \mathrm{E}, 127 \mathrm{~m}, 13 . i i i .2011$, T. Chaiwong (CMPH); $10^{7}$, Ubon Ratchathani Prov., Warinchamrap, School cafeteria, $15^{\circ} 11^{\prime} 33.212^{\circ} \mathrm{N}, 104^{\circ} 50^{\prime} 6.756^{\circ} \mathrm{E}, 126 \mathrm{~m}, 12$. iii.2011, T. Chaiwong (CMPH); $30^{\top}$, Ubon Ratchathani Prov., Warinchamrap, Fresh-food market, $15^{\circ} 10^{\prime} 40.369^{\circ} \mathrm{N}, 104^{\circ} 52^{\prime} 20.981^{\circ}$ E, 121 m, 21.v.2011, 11.vi.2011, T. Chaiwong (CMPH); 60 , Ubon Ratchathani Prov., Warinchamrap, Garbage piles, $15^{\circ} 11^{\prime} 52.340^{\circ} \mathrm{N}$, $104^{\circ} 52^{\prime} 10.045^{\circ} \mathrm{E}, 133 \mathrm{~m}, 12$. iii.2011, 2.iv.2011, 21.v.2011, 11. viii.2011, T. Chaiwong (CMPH). KO SAMUI I.: $10^{7}$, Surat Thani Prov., Ko Samui, N. Hinlad, A2, 20-21.iv.1988, H. Kurahashi (NIID); 4o , Surat Thani Prov., Ko Samui, Tong Yang, A3, 22.iv.1988, H. Kurahashi (NIID).

\section{Parasarcophaga hinglungensis Fan, 1964}

THAILAND: $10^{7}$, Chiang Mai Prov., Mae Taeng Dist., Mae Taeng Elephant Camp, 345 m, 14.xi.2011, H. Kurahashi (IDD).

\section{Parasarcophaga hirtipes (Wiedemann, 1830)}

THAILAND: $10^{7}$, Lampang Prov., Hang Chat Dist., Doi Khun Tan, 523 m, 8.xii.2007, H. Kurahashi (IDD); $2 \sigma^{7}$, Chiang Rai Prov., Pa Tuena Dist., Mae Chan, Huai Mahin Fon fall, 500 m, 5.xii.2007, H. Kurahashi (IDD); 50', Ubon Ratchathani Prov., Muang, Paddy field, $15^{\circ} 18^{\prime} 2.562^{\circ} \mathrm{N}, 104^{\circ} 52^{\prime} 50.035^{\circ} \mathrm{E}, 120 \mathrm{~m}$, 13.iii.2011, 22.v.2011, 12.vi.2011, T. Chaiwong (CMPH); 10 Ubon Ratchathani Prov., Warinchamrap, Paddy field, $15^{\circ} 8^{\prime} 21.388^{\circ}$ $\mathrm{N}, 104^{\circ} 53^{\prime} 52.218^{\circ} \mathrm{E}, 137 \mathrm{~m}, 11 . v i .2011$, T. Chaiwong (CMPH); $10^{\top}$, Ubon Ratchathani Prov., Muang, Restaurant, $15^{\circ} 16^{\prime} 29.668^{\circ} \mathrm{N}, 104^{\circ}$ $49^{\prime} 27.471^{\circ} \mathrm{E}, 132 \mathrm{~m}, 13$. iii.2011, T. Chaiwong (CMPH); $20^{\top}$, Ubon Ratchathani Prov., Muang, Restaurant, $15^{\circ} 16^{\prime} 29.668^{\circ} \mathrm{N}, 104^{\circ} 49^{\prime}$ $27.471^{\circ} \mathrm{E}, 132 \mathrm{~m}, 12 . v i .2011$, T. Chaiwong (CMPH); $20^{\top}$, Ubon Ratchathani Prov., Muang, School cafeteria, $15^{\circ} 16^{\prime} 29.668^{\circ} \mathrm{N}, 104^{\circ}$ $49^{\prime} 27.471^{\circ}$ E, 127 m, 22.v.2011, T. Chaiwong (CMPH); $40^{\top}$, Ubon Ratchathani Prov., Warinchamrap, School cafeteria, $15^{\circ} 11^{\prime} 33.212^{\circ}$ $\mathrm{N}, 104^{\circ} 50^{\prime} 6.756^{\circ} \mathrm{E}, 126 \mathrm{~m}, 11 . v i .2011$, T. Chaiwong (CMPH).

\section{Parasarcophaga idmais (Séguy, 1934)}

THAILAND: $10^{\nearrow}$, Chiang Mai Prov., Mae kuang dam, 20.vii.2000, S. Lertthamnongtham (DPCM); $2 \sigma^{\top}$, Chiang Rai Prov., Doi Suthep-Pui National Park, Sirindhorn Observatory, 817 m, 4-5. iii.2007, H. Kurahashi (IDD); $10^{\top}$, Chiang Mai Prov., Hang Dong Dist., Samoeng-Hang Dong rd., 390 m, 13.iii.2007, H. Kurahashi (IDD); $10^{\top}$, Chiang Rai Prov., Doi Saked-Ban Pang Deang, Nam Mae Kuang Bridge, 551 m, 7-8.iii.2007, H. Kurahashi (IDD) [10 $>\operatorname{DPCM}($ gift $)$.

\section{Parasarcophaga iwuensis Ho, 1934}

THAILAND: $10^{7}$, Chiang Mai Prov., Doi Sthep, 1,000 m, A6, 9-15.v.1988, H. Kurahashi (NIID). 
Parasarcophaga javana (Macquart, 1851)

Parasarcophaga doleschalli : Lopes al., 1977: 568. THAILAND: $10^{7}$, Chiang Mai Prov., Doi Inthanon, 2,600 m, A7, 10-12.v.1988, H. Kurahashi (NIID); $10^{\top}$, Chiang Mai Prov., Chom Thong, Doi Inthanon, 2,444 m, 18.34'27"N, 98.28'54"E, 12. xi.2009, H. Kurahashi (IDD) [1 $10^{\top}>$ NSMT(gift)]; $2 \sigma^{\top}$, Chiang Mai Prov., Doi Saket, Ban Pang Deang, Nam Mae Kuang Bridge, $551 \mathrm{~m}, 7-8$. iii.2007, H. Kurahashi (IDD) $\left[10^{\top}>\mathrm{UM}(\exp ), 10^{\top}>\right.$ NSMT(gift)].

\section{Parasarcophaga misera (Walker, 1849)}

THAILAND: $1 \sigma^{\top}$, Chiang Mai Prov., Mae aon, 16.xi.2000, S. Lertthamnongtham (DPCM); $10^{\text {T}}$, Tak Prov., $65 \mathrm{~km} \mathrm{NE}$ of Mae Sot, Ban Nai, 8.iv.1988, H. Kurahashi (NIID); $10^{\text {}}$, Khao Chamao, 27.viii.1986, Y. Wada (NIID); $30^{\nearrow}$, Chiang Mai Prov., Doi Inthanon, 1,300 m, A7, 10-12.v.1988, H. Kurahashi (NIID); $20^{7}$, Surat Thani Prov., nr Wat Nok, Khlong Sa, A4, 22.iv.1988, H. Kurahashi (NIID); $10^{7} 1$ 우, Chiang Mai Prov., Maung, HueyTueng Tao, lake, 9.iii.2007(10 $\left.10^{\top}\right)$, 24.vfii.2007(1우), H. Kurahashi (IDD); 1우, Chiang Mai Prov., Chom Thong, Doi Inthanon, 1,494 m, 18.30'52" $\mathrm{N}$, 98.31'33", 11.xi.2009, H. Kurahashi (IDD) [1우> UM(exp)]; 1우, Chiang Mai Prov., Doi Suthep-Pui National Park, Sirindhorn Observatory, 817 m, 27.vii.2007, H. Kurahashi (IDD); 1우, Chiang Mai Prov., Doi Saked-Ban Pang Deang, Nam Mae Kuang Bridge, 551 m, 7-8.iii.2007, H. Kurahashi (IDD); $30^{7}$, Ubon Ratchathani Prov., Muang, Paddy field, $15^{\circ} 18^{\prime} 2.562^{\circ} \mathrm{N}, 104^{\circ} 52^{\prime} 50.035^{\circ} \mathrm{E}, 120 \mathrm{~m}$, 22.v.2011, 12.vi.2011, T. Chaiwong (CMPH); $20^{\top}$, Ubon Ratchathani Prov., Warinchamrap, Paddy field, $15^{\circ} 8^{\prime} 21.388^{\circ} \mathrm{N}, 104^{\circ} 53^{\prime}$ 52.218 ${ }^{\circ}$ E, 137 m, 21.v.2011, 7.vii.2011, T. Chaiwong (CMPH); $20^{7}$, Ubon Ratchathani Prov., Warinchamrap, School cafeteria, $15^{\circ} 11^{\prime}$ $33.212^{\circ} \mathrm{N}, 104^{\circ} 50^{\prime} 6.756^{\circ} \mathrm{E}, 126 \mathrm{~m}, 11 . v i .2011$, T. Chaiwong (CMPH); $10^{\gamma}$, Ubon Ratchathani Prov., Warinchamrap, Garbage piles, $15^{\circ} 11^{\prime} 52.340^{\circ} \mathrm{N}, 104^{\circ} 52^{\prime} 10.045^{\circ} \mathrm{E}, 133 \mathrm{~m}, 21 . v .2011, \mathrm{~T}$. Chaiwong (CMPH). KO SAMUI I.: $40^{\nearrow}$, Surat Thani Prov., Ko Samui, N. Hinlad, A2, 20-21.iv.1988, H. Kurahashi (NIID)

\section{Parasarcophaga scopariiformis (Senior-White, 1927)} THAILAND: $2 \sigma^{7}$, Surat Thani Prov., nr wat Nok, Khlong Sa, A4, 22.iv.1988, H. Kurahashi (NIID); $4 \sigma^{\top}$, Tak Prov., $80 \mathrm{~km} \mathrm{NW}$ of Mae Sot, Mae Rayang, 9.iv.1988, H. Kurahashi (NIID); $10^{7}$, Tak Prov., 65 km NE of Mae Sot, Ban Mai, 8.iv.1988, H. Kurahashi (NIID); $40^{\top} 2$ 우, Chiang Mai Prov., Hang Dong Dist., T. Nongkaeo, $18.40^{\prime} 52^{\prime \prime} \mathrm{N}, 98.55^{\prime} 57^{\prime \prime} \mathrm{E}, 360 \mathrm{~m}$, 9.iii.2011, H. Kurahashi (IDD) $\left[20^{\top}>\mathrm{UM}\left(\right.\right.$ exp), $10^{\top}>\operatorname{DPCM}($ gift) $]$; $10^{\top}$, Chiang Mai Prov., Doi Suthep-Pui National Park, Sirindhorn Observatory, 817 m, 11.iii.2011, H. Kurahashi (IDD); 40 , Chiang Mai Prov., Saraphi Dist., T. Thawangtan, $18.43^{\prime} 08^{\prime \prime} \mathrm{N}, 98.59^{\prime} 13^{\prime \prime} \mathrm{E}, 315 \mathrm{~m}, 9$. iii.2011, H. Kurahashi (IDD); $10^{\nearrow}$, Chiang Mai Prov., Mae Taeng Dist., Mae Taeng Elephant Camp, 345 m, 14.xi.2011, H. Kurahashi (IDD); $10^{7}$, Chiang Mai Prov., Doi Suthep-Pui National Park, Sirindhorn Observatory, 817 m, 11.iii.2011, H. Kurahashi (IDD); $4 \sigma^{\urcorner} 2$ 우, Chiang Mai Prov., Hang Dong Dist., T. Nongkaeo, $18.40^{\prime} 52^{\prime \prime} \mathrm{N}, 98.55^{\prime} 57^{\prime \prime} \mathrm{E}, 360 \mathrm{~m}$, 9.iii.2011, H. Kurahashi (IDD) [20' $>\mathrm{UM}(\exp ), 1 \sigma^{\top}>\mathrm{DPCM}\left(\right.$ gift) ]; $2 \sigma^{\top}$, Ubon Ratchathani Prov., Muang, Paddy field, $15^{\circ} 18^{\prime} 2.562^{\circ} \mathrm{N}, 104^{\circ} 52^{\prime} 50.035^{\circ} \mathrm{E}, 120 \mathrm{~m}$, 23.i.2011, 12.vi.2011, T. Chaiwong (CMPH).
Parasarcophaga taenionota (Wiedemann, 1819)

THAILAND: $2 \sigma^{\top}$, Surat Thani Prov., nr Wat Nok, Khlong Sa, A4, 22.iv.1988, H. Kurahashi (NIID); 250', Chiang Mai Prov., Doi Sthep, 1,000 m, A6, 9-15.v.1988, H. Kurahashi (NIID); 120', Chiang Mai Prov., Doi Inthanon, 1,300 m, A7, 10-12.v.1988, H. Kurahashi (NIID); $20^{\top}$, Chiang Mai Prov., Doi Saked-Ban Pang Deang, Nam Mae Kuang Bridge, 551 m, 7-8.iii.2007, H. Kurahashi (IDD); 10 $\sigma^{\nearrow}$, Chiang Mai Prov., Chom Thong, Doi Inthanon, Ban Mae Klang, 924 m, 12.iii.2007, H. Kurahashi (IDD) $\left[10^{\top>}\right.$ BPBM(gift)]; $2 \sigma^{\gamma}$, Chiang Rai Prov., Pa Tuena Dist., Mae Chan, Huai Mahin Fon fall, 500 m, 5.xii.2007, H. Kurahashi (IDD) [20 $>$ ZMUC(gift)]; $1 \sigma^{\top}$, Chiang Mai Prov., Maung, HueyTueng Tao, lake side, 9.iii.2007, H. Kurahashi (IDD) [1 $10^{\top}>\mathrm{BMNH}$ (gift)]; 10 $10^{7}$, Chiang Rai Prov., Pa Tuena Dist., Mae Chan, Laan Tong resort, 449 m, 4.xii.2007, H. Kurahashi (IDD) $\left[10^{\top}>\operatorname{DPCM}\right.$ (gift)]. KO SAMUI I.: $10^{\top}$, Surat Thani Prov., Ko Samui, Tong Yang, A3, 22. iv.1988, H. Kurahashi (NIID); 20', Surat Thani Prov., Ko Samui, N. Hinlad, A2, 20-21.iv.1988, H. Kurahashi (NIID).

\section{Parasarcophaga yunnanensis Fan, 1964}

THAILAND: 20 $\sigma^{7}$, Chiang Mai Prov., Doi Sthep, 1,000 m, A6, 9-15.v.1988, H. Kurahashi (NIID); 17ð', Chiang Rai Prov., Pa Tuena Dist., Mae Chan, Huai Mahin Fon fall, 500 m, 5.xii.2007, H. Kurahashi (IDD) $\left[70^{7}>\mathrm{BMNH}\right.$ (gift), $70^{x}>\mathrm{BPBM}$ (gift), $30^{x}>$ ZMUC(gift)]; 4$\sigma^{7}$, Lampang Prov., Hang Chat Dist., Doi Khun Tan rest area, $523 \mathrm{~m}$, 28.vii.2007, H. Kurahashi (IDD) [10 $\left[0^{\top}>\right.$ $\operatorname{DPCM}$ (gift)]; 12 $\sigma^{\top}$, Lampang Prov., Hang Chat Dist., Doi Khun

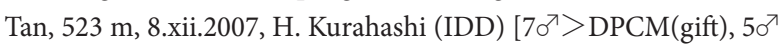
$>$ ZMUC(gift)]; 4疋, Chiang Mai Prov., Doi Suthep-Pui National

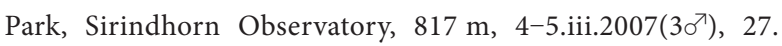
vii.2007(1 $\left.10^{\top}\right)$, H. Kurahashi (IDD); $3 \sigma^{\top}$, Chiang Mai Prov., Hang Dong Dist., Samoeng-Hang Dong rd., 390 m, 9.iii.2007, H. Kurahashi (IDD).

*Robineauella anchoriformis (Fan, 1964) THAILAND: $10^{\top}$, Chiang Mai Prov., Doi Suthep-Pui National Park, Doi Pui Summit-Sun Khu, 1,587 m, 4.iii.2007, T. Chaiwong (IDD).

Robineauella coei Rohdendorf, 1966

THAILAND: $10^{\top}$, Chiang Mai Prov., Doi Suthep-Pui National Park, Sun Khu, Doi Pui Summit, 1,587 m, 4.iii.2007, K. Moophayak (IDD) $\left[10^{\top}>\right.$ NSMT(gift)]; $10^{\nearrow}$, Chiang Mai Prov., Doi Pui Summit-Sun Khu, Doi Suthep-Pui National Park, 1,587 m, 4.

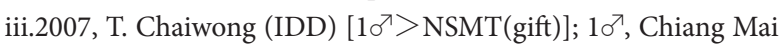
Prov., Muang, Doi Suthep-Pui Mount, Tham Phra Leusri, 950 m, 11.iii.2011, H. Kurahashi (IDD).

*Robineauella pseudoscoparia (Kramer, 1911) THAILAND: $10^{7}$, Chiang Mai Prov., Doi Suthep-Pui National Park, Doi Pui Summit-Sun Khu, 1,587 m, 4.iii.2007, T. Chaiwong (DPCM); $10^{\top}$, Chiang Mai Prov., Doi Suthep-Pui National Park, Doi Pui Summit-Sun Khu, 1,587 m, 4-5.iii.2007, H. Kurahashi (IDD); $10^{\top}$, Chiang Mai Prov., Muang, Doi Suthep-Pui Mount, Tham Phra Leusri, 950 m, 11.iii.2011, H. Kurahashi (IDD); 40 Chiang Mai Prov., Saraphi Dist., T. Thawangtan, $18.43^{\prime} 08^{\prime \prime} \mathrm{N}$, 98.59'13"E, 315 m, 9.iii.2011, H. Kurahashi (IDD); 10', Chiang 
Mai Prov., Muang, Doi Suthep-Pui Mount, Tham Phra Leusri, 950 m, 11.iii.2011, H. Kurahashi (IDD).

\section{Robineauella thailandica sp. nov.}

\section{Rosellea khasiensis (Senior-White, 1924)}

THAILAND: $20^{7}$, Chiang Mai Prov., Doi Inthanon, 1,300 m, A7, 10-12.v.1988, H. Kurahashi (NIID).

\section{Seniorwhitea princeps (Wiedemann, 1830)}

THAILAND: $4 \sigma^{7}$, Tak Prov., $80 \mathrm{~km}$ NW of Mae Sot, Mae Rayang, 9.vi.1988, H. Kurahashi (NIID); $2 \sigma^{\top}$, Tak Prov., 65 km NE of Mae Sot, Ban Mai, 8.vi.1988, H. Kurahashi (NIID); 12ð, Chiang Mai Prov., Doi Sthep, 1,000 m, A6, 9-15.v.1988, H. Kurahashi (NIID); $130^{7}$, Chiang Mai Prov., Doi Inthanon, 1,300 m, 10-12.v.1988, H. Kurahashi (NIID); 10', Chiangmai Prov., Chiangmai, 9.ix.1989, H. Kurahashi (NIID); 40 ${ }^{\top}$, Chiang Mai Prov., Doi Suthep-Pui National Park, Headquarters area, 1,075 m, 4-5.iii.2007, H. Kurahashi (IDD); $10^{7}$, Chiang Mai Prov., Maung, HueyTueng Tao, lake side, 9.iii.2007, H. Kurahashi (IDD); 10 ' Chiang Mai Prov., Chom Thong, Doi Inthanon, Ban Mae Klang, 924 m, 12.iii.2007, H. Kurahashi (IDD); $50^{\top}$, Chiang Mai Prov., Hang Dong Dist., Samoeng-Hang Dong rd., 390 m, 13.iii.2007, H. Kurahashi (IDD); 1207, Chiang Mai Prov., Doi Saked-Ban Pang Deang, $551 \mathrm{~m}, 7-8$. iii.2007, H. Kurahashi (IDD) $\left[70^{7}>\operatorname{DPCM}(\right.$ gift $), 50^{7}>$ $\mathrm{CMNH}$ (gift)]; $2 \sigma^{\top}$, Chiang Mai Prov., Muang, Doi Suthep-Pui,

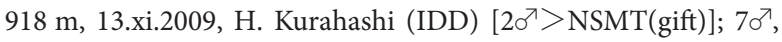
Chiang Mai Prov., Doi Saked, Ban Pangdoeng, 508 m, 25.vii.2007, H. Kurahashi (IDD) $\left[70^{\top}>\mathrm{DPCM}\right.$ (gift)]; $3 \sigma^{\top}$, Chiang Mai Prov., Chom Thong, Doi Inthanon, 924 m, 12.iii.2007, H. Kurahashi

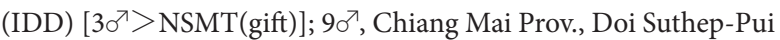
National Park, Sirindhorn Observatory, 817 m, 27.vii.2007, H.

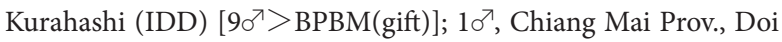
Saked, Doi Nang Kaew, 1,010 m, 26.vii.2007, H. Kurahashi (IDD) $\left[10^{\top}>\mathrm{BMNH}\right.$ (gift)]; 60 7 , Lampang Prov., Hang Chat Dist., Doi Khun Tan, 523 m, 8.xii.2007, H. Kurahashi (IDD) $\left[60^{\top>}\right.$ ZMUC(gift)]; $4 \sigma^{\gamma}$, Chiang Mai Prov., Doi Saket, Ban Pang Deang, Nam Mae Kuang Bridge, 551 m, 7-8.iii.2007, H. Kurahashi (IDD)

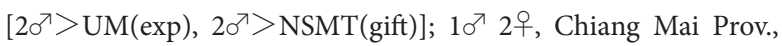
Doi Saket, Doi Nang Kaew, 1,016 m, 10.iii.2011, H. Kurahashi (IDD); 1우, Chiang Mai Prov., Doi Saket, Doi Nang Kaew, 972 m, 7-8.iii.2007, H. Kurahashi (IDD) [1우>NSMT(gift)]; 30 , Chiang Mai Prov., Doi Saket, Doi Nang Kaew, 1,142 m, 19.03'53"N, 99.22' 34"E, 14.xi.2009, H. Kurahashi (IDD) [30 $70^{7}>\operatorname{NSMT}$ (gift)]; 30 Chiang Mai Prov., Maung, HueyTueng Tao, lake side, 3.xii.2007, H. Kurahashi (IDD) $\left[3 \sigma^{\top}>\mathrm{BMNH}\left(\right.\right.$ gift)]; $2 \sigma^{\top}$, Chiang Rai Prov., Pa Tuena Dist., Mae Chan, Laan Tong resort, 449 m, 4.xii.2007, H. Kurahashi (IDD) $\left[20^{7}>\mathrm{BMNH}\right.$ (gift)]; $10^{\gamma}$, Chiang Mai Prov., Muang, Doi Suthep-Pui Mount, Tham Phra Leusri, $950 \mathrm{~m}$, 11.iii.2011, H. Kurahashi (IDD) $\left[10^{\top}>\mathrm{UM}(\exp )\right]$; 1우, Chiang Mai Prov., Hang Dong Dist., T. Nongkaeo, $18.40^{\prime} 52^{\prime \prime} \mathrm{N} 98.55^{\prime} 57^{\prime \prime}$ E, 360 m, 9.iii.2011, H. Kurahashi (IDD); 10', Chiang Rai Prov., Pa Tuena Dist., Mae Chan, Huai Mahin Fon fall, 500 m, 5.

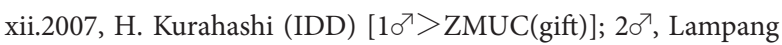
Prov., Hang Chat Dist., Doi Khun Tan rest area, 523 m, 28. vii.2007, H. Kurahashi (IDD) [20 $>$ BMNH (gift)]; $10^{\Upsilon} 2$ 우, Chiang Mai Prov., Doi Saket, Doi Nang Kaew, 1,016 m, 10.iii.2011, H.
Kurahashi (IDD); $10^{\nearrow}$, Chiang Mai Prov., Muang, Doi Suthep-Pui Mount, Tham Phra Leusri, 950 m, 11.iii.2011, H. Kurahashi (IDD) $\left[10^{\top}>\mathrm{UM}(\exp )\right] ; 1$ 우, Chiang Mai Prov., Hang Dong Dist., T. Nongkaeo, 18.40'52"N, 98.55'57"E, 360 m, 9.iii.2011, H. Kurahashi (IDD); 1 우, Chiang Mai Prov., Mae Taeng Dist., Mae Taeng Elephant Camp, 345 m, 14.xi.2011, H. Kurahashi (IDD); 10', Chiang Mai Prov., Doi Suthep-Pui Mt., Siridhorn Observatory, 817 m, 15.xi.2011, H. Kurahashi (IDD); $10^{\top}$, Ubon Ratchathani Prov., Muang, School cafeteria, $15^{\circ} 16^{\prime} 29.668^{\circ} \mathrm{N}, 104^{\circ} 49^{\prime} 27.471^{\circ} \mathrm{E}$, 127 m, 12.vi.2011, T. Chaiwong (CMPH); 20 $\sigma^{\top}$, Ubon Ratchathani Prov., Warinchamrap, Paddy field, $15^{\circ} 8^{\prime} 21.388^{\circ} \mathrm{N}, 104^{\circ} 53^{\prime} 52.218^{\circ}$ E, 137 m, 7.vii.2011, 22.i.2011, T. Chaiwong (CMPH).

\section{Sinonipponia hainanensis (Ho, 1936)}

THAILAND: $3 \sigma^{\top}$, Tak Prov., 80 km NW of Mae Sot, Mae Rayang, 9.iv.1988, H. Kurahashi (NIID); 10', Tak Prov., 65 km NE of Mae Sot, Ban Mai, 8.iv.1988, H. Kurahashi (NIID).

Sinonipponia komi Kurahashi \& Sukontason, 2004 THAILAND: $10^{\top}$, Ubon Ratchathani Prov., Warinchamrap, Paddy field, $15^{\circ} 8^{\prime} 21.388^{\circ} \mathrm{N}, 104^{\circ} 53^{\prime} 52.218^{\circ} \mathrm{E}, 137 \mathrm{~m}, 21 . v .2011$, T. Chaiwong $(\mathrm{CMPH})$.

\section{ACKNOWLedgements}

The present authors wish to express their sincere gratitude to the late Dr. R. Kano, Leader of Project, Professor emeritus, and Dr. S. Shinonaga, Tokyo Medical and Dental University, Japan for offering an opportunity to study the fly fauna of Thailand. For the recent field surveys in Chiang Mai area, the authors thank to Prof. Dr. K. Sukontason and Prof. Dr. K. L. Sukontason, Department of Parasitology, Faculty of Medicine, Chiang Mai University for offering an opportunity to study the sarcophagid fly fauna of Thailand. We would like to thank Dr. N. Bunchu, Department of Microbiology and Parasitology, Faculty of Medical Science, Naresuan University, Thailand for the field surveys and kind assistance. The authors thank to Dr. K. Moophayak, Mahidol University Nakhonsawan Campus, Nakhonsawan, Thailand, for his kind cooperation and assistance during the field survey. The second author (TC) was financially supported by the Thailand Research Fund through the Royal Golden Jubilee Ph. D. Programm (Grant No. PHD/0113/2547) and (MRG 5380283).

\section{REFERENCES}

Chaiwong, T., Sukontason, K. and Sukontason, K. L. 2009. Two new species of Sarcophaga s. lat. from Thailand with a key to species (Diptera: Sarcophagidae). J. Med. Entomol., 46: 986-993.

Fan, Z. 1965. Key to the Common Flies of China. 330 pp., Science Press, Beijing (in Chinese).

Kurahashi, H. 1970. Studies on the Calypterate Muscoid flies from Japan VII. Revision of the subfamily Miltogramminae (Diptera, Sarcophagidae). Konty $\hat{u}, 38$ : 93-116.

Kurahashi, H. and Sukontason, K. L. 2004. A new species of Sinonipponia (Diptera: Sarcophagidae) from Thailand. Med. Entomol. Zool., 55: 191-193. 
Kurahashi, H. and Tan, S. H. 2009. The sarcophagid flies from Peninsular Malaysia (Diptera: Sarcophagidae). Med. Entomol. Zool., 60: 283-296.

Lopes, H. de Souza, Kano, R., Shinonaga, S. and Kurahashi, H. 1977. Family Sarcophagidae. 557-583 pp. In: Delfinado, M. D. and D. E. Hardy (eds.); A catalog of the Diptera of the Oriental Region. Volume III. Suborder Cyclorrhapha (excluding Division Aschiza), 557-583. University Press of Hawaii, Honolulu.

Nandi, B. C. 2002. Diptera. Family Sarcophagidae. In: The Fauna of India and the Adjacent Countries. Vol. X. xxiv +608 pp., Zoological Survey of India, Kolkata.

Pape, T., 1996. Catalogue of the Sarcophagidae of the World (Insecta: Diptera). Mem. Ent., Internat., 8: 1-558.

Pape, T. and Bänziger, H. 2000. Two new species of Sarcophaga (Diptera: Sarcophagidae) among pollinators of newly discovered Sapria ram (Rafflesiaceae). Raffles Bull. Zool., 48: 201-208.

Pape, T. and Bänziger, H. 2003. Three new species of Sarcophaga Meigen found during ecological studies on flesh flies (Diptera:
Sarcophagidae) in Thailand. Entomol. Sci., 6: 49-56.

Povolný, D. and Verves, Yu. 1997. The flesh-flies of Central Europe (Insecta, Diptera, Sarcophagidae). Spixiana, suppl. 24: 1-260.

Rohdendorf, B. B. 1937. Fam. Sarcophagidae. Fauna USSR, 19: xv + $1-501+[1]$ (In Russian with German summary)

Rohdendorf, B. B. 1966. Diptera from Nepal. Sarcophagidae. Bull. Br. Mus. Nat. Hist. Ser. B. Ent., 17: 457-464.

Senior-White, R. A., Aubertin, D. and Smart, J. 1940. Diptera. Family Calliphoridae. In: The fauna of British India, including the reminder of the Oriental Region. Vol. VI. xiii +288 pp., Taylor and Francis, Ltd., London.

Sugiyama, E., Shinonaga, S. and Kano, R. 1990. Sarcophagine flies from Malaysia and Singapore with the descriptions of six new species (Diptera, Sarcophagidae). Jpn. J. Sanit. Zool., 41: 81-91.

Tumrasvin, W. and Kano, R. 1979. Studies on medically important flies in Thailand VI. Report on 48 species of sarcophagid flies, including the taxonomic keys (Diptera: Calliphoridae). Bull. Tokyo Med. Dent. Univ., 26: 149-179. 